\title{
Numerical re-creation of multi-directional waves in a circular basin using a particle based method
}

DOI:

10.1016/j.oceaneng.2020.107446

\section{Document Version}

Accepted author manuscript

Link to publication record in Manchester Research Explorer

\section{Citation for published version (APA):}

Kanehira, T., Mutsuda, H., Draycott, S., Taniguchi, N., Takuji, N., Doi, Y., \& Ingram, D. M. (2020). Numerical recreation of multi-directional waves in a circular basin using a particle based method. Ocean Engineering. https://doi.org/10.1016/j.oceaneng.2020.107446

\section{Published in:}

Ocean Engineering

\section{Citing this paper}

Please note that where the full-text provided on Manchester Research Explorer is the Author Accepted Manuscript or Proof version this may differ from the final Published version. If citing, it is advised that you check and use the publisher's definitive version.

\section{General rights}

Copyright and moral rights for the publications made accessible in the Research Explorer are retained by the authors and/or other copyright owners and it is a condition of accessing publications that users recognise and abide by the legal requirements associated with these rights.

\section{Takedown policy}

If you believe that this document breaches copyright please refer to the University of Manchester's Takedown Procedures [http://man.ac.uk/04Y6Bo] or contact uml.scholarlycommunications@manchester.ac.uk providing relevant details, so we can investigate your claim.

\section{OPEN ACCESS}


1

2

3

\title{
Numerical re-creation of multi-directional waves in a circular basin using a particle based method
}

\author{
Taiga Kanehira ${ }^{\mathrm{a}}$, Hidemi Mutsuda ${ }^{\mathrm{b}, *}$, Samuel Draycott ${ }^{\mathrm{c}}$, Naokazu Taniguchi ${ }^{\mathrm{b}}$, \\ Takuji Nakashima ${ }^{\mathrm{b}}$, Yasuaki Doi ${ }^{\mathrm{b}}$, David Ingram ${ }^{\mathrm{d}}$ \\ ${ }^{a}$ Department. of Transportation and Environmental Systems, Graduate School of \\ Engineering, Hiroshima University, Japan \\ ${ }^{b}$ Academy of Science and Technology, Mechanical Engineering/Integrated Engineering Unit, \\ Graduate School of Engineering, Hiroshima University, Japan \\ ${ }^{c}$ School of Mechanical, Aerospace and Civil Engineering, The University of Manchester, \\ Manchester, UK, M60 1QD \\ ${ }^{d}$ Institute for Energy Systems, School of Engineering, The University of Edinburgh, \\ Edinburgh, EH9 3JL, UK
}

\begin{abstract}
Numerical wave basins are a powerful tool for the study of fluid-structure interaction (FSI) problems in coastal and ocean engineering fields. Once well validated, numerical wave basins can offer significant advantages over experimental testing in terms of the number and type of available data-streams, and the associated cost. However, conventional numerical wave tanks tend to be limited to the generation of long-crested conditions, and struggle to properly model large fluid deformations along with wave-induced floating body motions and pressure distribution on structures. Here, for the first time we create, and validate, the short-crested wave fields in a circular wave basin, using a particlebased method to solve the full 3D Navier-Stokes equation. The model is based on the FloWave facility at the University of Edinburgh: the geometry enables the generation of multi-directional waves, and the particle-based-approach enables large fluid deformations to be automatically modelled. Multi-directional waves are generated with differing values of steepness and directional spreading, and simulated surface elevations are compared to experimentally-obtained data.
\end{abstract}

\footnotetext{
* Corresponding author

Email address: mutsuda@hiroshima-u.ac.jp (Hidemi Mutsuda)

${ }^{1}$ 1-4-1 Kagamiyama Higashi-Hiroshima, 739-8527, Japan
}

Preprint submitted to Journal of Ocean Engineering 
Acceptable agreement is found between measured and modelled surface elevation values. Better performance is confirmed for lower-steepness wave cases; where $r^{2}$ values of greater than 0.7 are found for all cases, and errors in significant wave height range between $-8 \%$ to $+1 \%$. Greater under-production is found for higher-steepness conditions with a $-10 \%$ to $-16 \%$ error in significant wave height. It is concluded that a greater number of particles with smaller radius is required to accurately capture the smaller higher frequency disturbances. It is suggested, however, that as the lower-frequency components are well produced for all cases that the model is already suitable for the majority of FSI problems in both uni- and multi-directional wave fields.

Keywords: Particle based method, SPH, DualSPHysics, Short-crested waves, Circular basin, FloWave

\section{Introduction}

The development of ocean energy converters (OECs), such as fixed or floating offshore wind turbines (OWTs), wave energy converters (WECs) and tidal energy converters (TECs), has attracted significant attention due to a growing desire to find alternative low-carbon energy sources (e.g. [1, 2]). A variety of experimental and numerical methods $([3,4,5,6])$ have been used to further the understanding of the interaction between the ocean environment and OECs. Understanding how complex, short-crested, sea conditions influence the dynamics and the electricity generation capacity of OECs is important because devices installed in the open-ocean will be regularly exposed to multi-directional wave fields.

Experimental simulation of realistic directional sea states are made possible by multi-directional wave basins; typically consisting of wavemakers along one or two sides of a rectangular basin. Circular basins, which completely remove limits on directionality, include the circular AMOEBA basin at Osaka University, comprising a 50-element-absorbing wavemaker of $1.6 \mathrm{~m}$ in diameter and with a water depth of $0.25 \mathrm{~m}$ [7], as well as a $15 \mathrm{~m}$ deep-sea basin with a 

128-element-absorbing wavemaker at the National Maritime Research Institute, Tokyo, Japan (see [8]). The FloWave Ocean Energy Research Facility (FloWave) at the University of Edinburgh in Scotland (https://flowave.eng.ed.ac.uk/) is the largest of the circular basins with a $25 \mathrm{~m}$ diameter. FloWave, depicted in Fig. 1, was constructed in 2013 and is the first basin in the world designed for the generation of waves and currents at arbitrary angles. This enables the creation of severe multi-directional design conditions [9, 10]. FloWave comprises 168 active-absorbing force-feedback hinged-flap-type paddles, which generate monochromatic waves and full-spectrum multi-directional waves. International research conducted on the FloWave basin ( e.g. $[11,12,13,14,15,16])$ has included the investigation of OEC performance and loading under realistic sea conditions prior to installation in test locations, such as the Orkney Islands (UK) and Nagasaki (Japan).

In line with experimental testing, numerical tanks have also been developed and utilised for designing OECs and studying their performance. These tanks are based on a variety of numerical techniques. A common approach is to solve the Navier-Stokes (NS) equation using a finite difference method. Turnbull et al. [17] developed a numerical wave tank based on a transformed finite element solution of the Laplace's equation to calculate and validate the generation of focussed wave groups. Regular and extreme wave conditions were calculated using the NS equation with a constrained interpolation profile (CIP) method in a two-dimensional (2D) numerical tank that was developed in [18]. Furthermore, Higuera et al. [19] produced a three-dimensional (3D) numerical wave generation model with moving boundaries using the IHFOAM simulator [20]. Windt et al. [21] developed a 3D numerical tank using the OpenFOAM software and computed floating motions by solving the full 3D NS equation.

It is difficult to simulate violent free surfaces using these grid based methods, as mesh deformation becomes increasingly large and simulations fail due to mesh overlapping; however, particle-based methods, a meshless Lagrangian approach, such as smoothed particle hydrodynamics (SPH) [22] and moving particle semi-implicit (MPS) [23], demonstrate remarkable advantages in solving 
fluid-structure interactions with violent flow. A recent review of particle-based methods can be found in [24]. These research works are being increasingly used for coastal and ocean engineering problems (e.g. [25, 26, 27, 28, 29]) including the related work on Incompressible SPH [30] and MPS. Based on the particlebased methods, there is no need to employ special treatment for the free surfaces or to calculate the advection term. Moreover, the particle methods facilitate the application of complex boundaries. Thus, many coastal and ocean engineering researchers have developed numerical tanks using the particle-based methods. Molteni et al. [31] simulated floating body motion with regular waves in a 2D numerical tank. Using the open-source code DualSPHysics [32], the simulation of waves with complex breakwater geometries in a 3D numerical tank was achieved in [33]. Crespo et al. [34] studied the effects of mooring systems on a floating oscillating water column (OWC), and Canelas et al. [35] coupled the Project Chrono library (an open-source multi-physics simulation engine) with the DualSPHysics code and applied the method in several simulations to explore WEC and TEC responses. Gunn et al. [36] simulated the interaction between surface waves and floating tethered buoy in uni-directional sea states. Masuda et al. [37] carried out the performance analysis of the fixed type OWC-WEC using improved MPS method. Brito et al. [38] also investigated the motion of oscillationg wave surge converter devices coupling Chrono with SPH. Kanehira et al. [39] have developed a circular wave basin using a particle-based method, DualSPHysics [32] open-source code, and validated long-crested regular and irregular wave conditions, and a concentric focused wave with experimental data recorded at FloWave.

Although a significant number of numerical wave tanks have been developed, many of these tanks numerically generate uni-directional (long-crested) waves. In contrast, numerical model of multi-directional short-crested waves are scarce and remain one of the challenging topics. To the author's best knowledge, several studies for short-crested (multi-directional) wave simulation are found as follows. Fuhrman et al. [40] investigated fully nonlinear evolution of short-crested wave instabilities in deep water solving nonlinear Boussinesq-type 
formulation. Ren et al. [41] developed a numerical circular wave basin based on grid based finite volume method to solve the NS equations, showing both longcrested and two crossing waves. Wei et al. [42] utilised the GPUSPH [43] to generate short-crested waves based on a moving boundary and the synchronous wave train method [44]. In this way, these authors conducted numerical investigations on short-crested waves in the surf zone. Recently, Kirby et al. [45] investigated short-crested wave breaking using Boussinesq model FUNWAVETVD [46].

As mentioned above, the particle based method has the enabling potential to fully reproduce the FSI problems with breaking waves, however, this study presented here focuses on the re-creation of full 3D short-crested waves generated and absorbed by 168 hinged-flap-type paddles using a particle based method implemented in DualSPHysics. First, in Sections 2 and 3, we will explain the experimental and numerical method, respectively, including their setup, as well as the mathematical background for short-crested wave generation. This is followed by a presentation and discussion of the model outputs and their subsequent validation in Section 4. Concluding remarks and comments on potential future studies are presented in Section 5 .

\section{Experimental Methods}

All experimental tests presented in this paper were conducted at the aforementioned circular FloWave basin, University of Edinburgh (Fig. 1). The basin is $25 \mathrm{~m}$ in diameter with a fixed water depth of $2 \mathrm{~m}$. In order to validate the generation of multi-directional waves in the numerical model, several sea states were generated as detailed in Section 2.1. This enables the model performance to have different values of wave steepness and spreading. The measured water surface elevations at gauge positions (presented in Section 2.2) were also utilised in the model validation. 


\subsection{Test conditions}

The target specifications of the waves generated in FloWave are presented in Table 1. $H_{m 0}$ denotes the significant wave height and $T_{p}$ represents the peak period for the Pierson-Moskowitz (PM) spectra [47], specifically $H_{m 0}=0.15 \mathrm{~m}$ and $T_{p}=1.5 \mathrm{~s}$, as well as $H_{m 0}=0.075 \mathrm{~m}$ and $T_{p}=3.0 \mathrm{~s}$, respectively. This provided two peak steepness values (defined as $H_{m 0} / L_{p}$, where $L_{p}$ is the peak wavelength) of 0.043 and 0.0053 , respectively. For both of the spectra, four spreading values were utilised $(s=5,10,25$ and Inf., where Inf. represents long-crested waves). This yields the eight test cases defined in Table 1. The computational time is $64 \mathrm{~s}$ in all cases, because of the computational cost of running longer simulations in this numerical model. This enabled the effective validation of the generated wave conditions, but also had implications for the effective creation of the target directional spectra, as discussed in Section 2.3.

The eight wave specifications are visualised spectrally in Fig.4, where the frequency and directional energy distributions are shown. The full directional spectra are shown for Cases $1 \sim 4$.

\subsection{Test setup}

In order to validate the short-crested wave generation performance, free surface elevations were measured at various position close to the centre of the FloWave basin, as shown in Fig. 5. The wave gauges (WGs) are multiplexed two-wire resistance-type probes, which were set to measure surface elevations at a sampling frequency of $32 \mathrm{~Hz}$ in this experiment. Wave Gauges $1 \sim 8$ are positioned along the mean wave propagation direction $(x=0)$, with WG4 at the centre of the tank $(x, y)=0$. Additional gauges (9 and 10) were installed along different $x$-positions to capture a wider region of the basin. The expected experimental errors resulting from the wave gauges are detailed in Appendix A.

\subsection{Short-crested wave generation}

The classic definition of directional spectra, and indeed the method presented in Fig. 4, is described as a distribution of energy in wave direction for every 
wave frequency. This description was extended to short-crested wave generation using the following equation for the surface elevation at point $[x, y]$ at time $t$ :

$$
\eta(x, y, z, t)=\sum_{i=0}^{N} \sum_{j=0}^{M} A_{i j} \cos \left(k_{j}\left[x \cos \theta_{i}+y \sin \theta_{i}\right]-\omega_{j} t+\epsilon_{i j}\right)
$$

where $\eta$ is the water surface elevation, $M$ is the number of frequency components and $N$ is the number of directional components, and the sea surface is described as a double sum over these components (i.e. the double-summation method). However, this approach to short-crested wave generation results in phase-locking as waves of equal frequency but different directions interact [48]. This creates spatial interference patterns in wave amplitude and results in a non-ergodic wave fields that is highly undesirable for experimental testing. In order to avoid this effect, the single-summation method was used (as is applied exclusively at FloWave) whereby each frequency is attributed to a unique wave propagation direction $\theta_{j}$ as follows:

$$
\eta(x, y, z, t)=\sum_{j=0}^{M} A_{j} \cos \left(k_{j}\left[x \cos \theta_{j}+y \sin \theta_{j}\right]-\omega_{j} t+\epsilon_{j}\right)
$$

where $k_{j}$ is the wave number, $\omega_{j}$ denotes the angular frequency of the wave, $\epsilon_{j}$ is the phase of the wave and $A_{j}$ represents the wave amplitude of component $j$. The single-summation approach to directional sea state generation minimises phase locking but requires a large number of frequency components to achieve the desired directional distribution over a given frequency band (whether the whole frequency range or a subset of it). In FloWave, the number of frequency components is determined by the repeat time $\left(T_{R}\right)$ of the tests, such that $\delta F=1 / T_{R}$. Hence, the short repeat times $(64 \mathrm{~s})$ used in our experiments means that the full target directional spectra could not be attained. Nevertheless, these short run conditions can be effectively used for model validation, and they effectively represent a short segment of a full statistical representation of the target spectrum. As described in Section 2.3, the input spectra were defined using the single-summation method. In order to further visualise this approach and to define the input wave conditions, the 'fronts', defining the individual fre- 
quency components with specific amplitudes, phases and directions are shown for the eight wave cases in Fig 6.

\section{Numerical Method}

\subsection{SPH Implementation}

\subsubsection{SPH fundamentals}

Based on the authors previous work [39] using DualSPHysics [32], the SPH, Lagrangian mesh-free, particle based method that was proposed in [22] was employed to describe wave motion. In this way, continuum fluid flow could be discretised as calculation points called particles, which move in conjunction with fluid motion. Furthermore, physical quantities such as pressure, density and velocity components can be described for each particle by spatial interpolation between neighboring particles with a smoothing length.

The fundamental principle of the SPH method, as shown in Fig.2, is to approximate the physical quantity $\phi$ as follows:

$$
\phi(\mathbf{r})=\int_{\Omega} \phi\left(\mathbf{r}^{\prime}\right) W\left(\mathbf{r}-\mathbf{r}^{\prime}, h\right) d \mathbf{r}^{\prime}
$$

where $W$ is the kernel function, $h$ is the referenced length, $\mathbf{r}$ is the focused position vector, $\Omega$ is the referenced area with a radius to evaluate interaction between particles and $\mathbf{r}^{\prime}$ is the neighbouring position vector. In this study, we use the quintic Wendland kernel reported in [49] for all the work presented in this study that is expressed as:

$$
W\left(\mathbf{r}_{a}, h\right)=\alpha_{D}\left(1-\frac{q}{2}\right)^{4}(2 q+1), \quad 0 \leq q \leq 2
$$

where $q=r / h$ is given by the distance between any selected two particles $(r)$ divided by smoothing length $h$, and $\alpha_{D}$ is equal to $7 / 4 \pi h^{2}$ in $2 \mathrm{D}$, and $21 / 16 \pi h^{3}$ in $3 \mathrm{D}$. Eq. (3) can be converted into the discretization form that is represented by:

$$
\phi\left(\mathbf{r}_{a}\right)=\sum_{b=1}^{N} \phi\left(\mathbf{r}_{b}\right) W\left(\mathbf{r}_{b}-\mathbf{r}_{a}, h\right) V_{b}
$$


where $a$ is a focused position particle and $V_{b}$ is the volume of a neighbouring particle $b$, and $V_{b}=m_{b} / \rho_{b}$, where $m$ and $\rho$ represent the mass and density of particle $b$, respectively,

\subsubsection{Governing equations}

The incompressible fluid can be described by the continuity and the momentum conservation:

$$
\begin{gathered}
\frac{D \rho}{D t}+\rho \nabla \cdot \mathbf{v}=0 \\
\frac{D \mathbf{v}}{D t}=-\frac{1}{\rho} \nabla P+\mathbf{g}+\nu_{0} \nabla^{2} \mathbf{v}+\frac{1}{\rho} \nabla \cdot \vec{\tau}
\end{gathered}
$$

where $D / D t$ denotes the Lagrangian time derivative, $\rho$ is the fluid density, $\mathbf{v}$ is the velocity vector, $\mathbf{g}$ is the gravitational acceleration, $P$ is the pressure, $\nu_{0}$ is the laminar kinematic viscosity and $\vec{\tau}$ is the SPS stress tensor. Using the SPH manner and following [50], Eqs. (6) and (7) represented as follow:

$$
\begin{array}{r}
\frac{d \rho_{a}}{d t}=\sum_{b} m_{b}\left(\mathbf{v}_{a}-\mathbf{v}_{b}\right) \cdot \nabla_{a} W_{a b}+\mathcal{D}_{a} \\
\frac{d \mathbf{v}_{a}}{d t}=-\sum_{b} m_{b}\left(\frac{P_{b}}{\rho_{b}^{2}}+\frac{P_{a}}{\rho_{a}^{2}}\right) \nabla_{a} W_{a b}+\mathbf{g} \\
+\sum_{b} m_{b}\left(\frac{4 \nu_{0} r_{a b} \cdot \nabla_{a} W_{a b}}{\left(\rho_{a}+\rho_{b}\right)\left(r_{a b}^{2}+\eta^{2}\right)}\right)\left(\mathbf{v}_{a}-\mathbf{v}_{b}\right) \\
\quad+\sum_{b} m_{b}\left(\frac{\vec{\tau}_{i j}^{b}}{\rho_{b}^{2}}+\frac{\vec{\tau}_{i j}^{a}}{\rho_{a}^{2}}\right) \nabla_{a} W_{a b}
\end{array}
$$

where $\eta^{2}=0.01 h^{2}$ and $\mathcal{D}_{a}$ in Eq. (8) represents a diffusive term, known as the delta-SPH scheme [31]. The delta-SPH coefficient used here is 0.1. In this study, the above technique is employed to reduce the pressure noise in the basin. In Eq.(9), the third term represents the laminar viscosity, as presented in [51], and the fourth term is the sub-particle scale (SPS) turbulence model firstly introduced by [52], and formulated in Weakly Compressible SPH by [50]. Following the latter study, we used the Smagorinsky constant (0.12). 


\subsubsection{Equation of state for pressure}

Assuming a weakly compressible fluid, pressure can be computed using an explicit numerical algorithm. Here, rather than solving Poisson's equation (an implicit method), an equation of state [Eq.(10)] that relates pressure to density was used to enable the reduction of computational costs;

$$
P=b\left[\left(\frac{\rho}{\rho_{0}}\right)^{\gamma}-1\right]
$$

In Eq.(10), where $\gamma=7$ and $b=c_{0}^{2} \rho_{0} / \gamma, \rho_{0}=1000 \mathrm{~kg} / \mathrm{m}^{3}$ is the referenced density and $c_{0}$ is the sound speed given by $c_{0}=\sqrt{\partial P / \partial \rho}$.

\subsubsection{Time stepping schemes}

Here, an explicit second-order time integration method (the symplectic scheme) was employed. This scheme has the following iteration steps known as the predictor [Eqs.(11) and (12)] and corrector stage [Eqs.(13) and (14)];

$$
\begin{aligned}
\mathbf{r}_{a}^{n+1 / 2} & =\mathbf{r}_{a}^{n}+\frac{\Delta t}{2} \mathbf{v}_{a}^{n} \\
\rho_{a}^{n+1 / 2} & =\rho_{a}^{n}+\frac{\Delta t}{2} D_{a}^{n} \\
\mathbf{v}_{a}^{n+1} & =\mathbf{v}_{a}^{n+1 / 2}+\frac{\Delta t}{2} \mathbf{F}_{a}^{n+1 / 2} \\
\mathbf{r}_{a}^{n+1} & =\mathbf{r}_{a}^{n+1 / 2}+\frac{\Delta t}{2} \mathbf{v}_{a}^{n+1}
\end{aligned}
$$

where the superscript $n$ indicates the time step, $\Delta t$ is the time increment for step $n$ and $\mathbf{F}$ consists of the pressure gradient term, diffusion term and gravitational acceleration term. The corrected density at each subsequent time step $(n+1)$, $d \rho_{a}^{n+1} / d t=D_{a}^{n+1}$, where $D$ is the divergence, can be computed using $\mathbf{v}_{a}^{n+1}$ and $\mathbf{r}_{a}^{n+1}$ computed by Eqs.(13) and (14) in the corrector stage. According to [53], variable time step $\Delta t$ is employed in this study.

\subsubsection{Boundary condition}

In this study, the dynamic boundary condition (DBC) is utilised for boundary condition; all paddles, that surrounding the circular wave basin, and fixed bottom floor (depicted in Figs. 8 and 9) consist of dynamic boundary particles. After [54] firstly proposed the DBC, a number of researchers have been 
employed this method for coastal/ocean engineering problems (e.g. [55, 56]), because of its simplicity of implementation, low computational cost, and easily application for complex geometry.

It should be noted that the DBC yields also the non-physical behaviour, in the vicinity of the boundary, namely, undesirable gaps between the fluid and boundary (including floating objects) particles, unstable pressure fields (e.g. see $[57,58])$. However, the geometry of numerical basin presented in this study has a complex boundary; 168 hinged-flap wave boards that will be controlled individually, thus, by considering its applicability to the complex boundary, we employ the DBC to all of following works and all wave paddles will be comprised of the dynamic boundary particles (DBP).

Fig. 3 is the sketch of the interaction between a fluid particle (blue thick line circle) and a number of boundary particles (orange thin line circle), showing a repulsion force (red arrow) acting on a fluid particle. Fluid particles will be subjected to the repulsion force, when approaching the DBP, and only if the distance between them becomes shorter than $2 h$. Generally, the forces exerted on the particles can be written by:

$$
\frac{D \mathbf{v}_{a}}{D t}=-\left(2 c^{2} \frac{W_{a b}}{\left(W_{a b}+W_{0}\right)^{2}}\right) \frac{\partial}{\partial \mathbf{r}_{a}} W_{a b}
$$

where $W_{0}=W_{a a}=W_{b b}, W_{0}=\rho_{0} / m$, subscript $a$ and $b$ denote a incoming fluid particle and dynamic boundary particles respectively. In other words, the repulsion mechanism would be numerically explained as follows; the local density of incoming particle a is increased following Eq. (10), that results in an increase of pressure term in Eq. (9) as pressure is defined by Eq. (10), thus incoming particle is subject to repulsion force (detailed results in [59]).

\subsection{FloWave Modelling}

The numerical FloWave basin model developed in [39] was utilised to reproduce short-crested wave fields. Figure 7 shows the surface configuration of a numerical wave basin with a $25 \mathrm{~m}$ diameter and $2 \mathrm{~m}$ water depth fluid domain, 
which utilises 168 hinged-flap-type wavemakers for wave generation and absorption. The wavemaker paddles are comprised of dynamic boundary particles as proposed in [60]. It should be noted that, unlike the real FloWave basin, the numerical basin has a flat bottom without inlet or outlet zone near the wavemakers for current circulation. Example particles distributions around wave paddles are depicted in Figs. 8 and 9. In order to reproduce the same wave conditions as those experimentally, the angles of each of the 168 wave paddles were recorded at the FloWave for each of the modelled sea states. These values are forced as the boundary conditions for the SPH simulation, and hence are implemented exactly as in the experiment. Fig. 10 shows the example of the recorded angle data exerting on paddles rotation in $\mathrm{SPH}$ model.

\subsection{Parametric study}

Before finalising the numerical parameters used in this study, we calculated a long-crested irregular wave case (represented in Table 1) for parametric study of this model with various numerical option implemented in DualSPHysics, such as kernel function, viscosity treatment and $h / d p$. All cases, $d_{p}$ was set to 0.03 $\mathrm{m}$.

Fig. 11 shows examples of the results. From Fig. 11(a) it is apparent that the Wendland kernel is superior to cubic spline kernel; the former is in phase with experimental data, however, the latter is out of phase. Regarding the viscosity treatment, the Laminar+SPS model shows better agreement with experimental, rather than Artificial model; the latter shows underestimated elevation results as shown in Fig. 11(b). This wave decay becomes noticeable for long time simulation, larger than $30 \mathrm{~s}$. In this study, we attempt to simulate short-crested wave fields for $64 \mathrm{~s}$ (that of [39] was $30 \mathrm{~s}$ ), and the laminar+SPS model is suitable to do that. Finally, we calculated three numerical tests, varying the value of $h / d_{p}, 1.7,2.1$ and 2.4, but taking the same Wendland kernel and Laminar+SPS viscosity model. In DualSPHysics, $h$ is determined as $h=\operatorname{coe} f h \sqrt{3 d_{p}{ }^{2}}$, where coefh denotes the coefficient of smoothing length, and the values ranged from 1.2 to 1.5 are recommended for wave propagation in DualSPHysics. In the 
calculation with varying $h / d_{p}$, the corresponding values of coefh are 1.0, 1.2 and 1.4, respectively. From Fig. 11(c), it is obvious that $h / d_{p}$ values of both 1.7 and 2.1 represents better results than that of 2.4, even though the corresponding value of coefh was set to 1.0 when $h / d_{p}=1.7$. However, it should be also noted that the resulting computational time of $h / d_{p}=2.1$ (2.4) was 1.4 (1.8) times longer comparing with that of $h / d_{p}=1.7$ using the same GPU machine (GeForce GTX $1080 \mathrm{Ti}$ ).

By considering the above results; the accuracy and the computational cost, we employed the Wendland Kernel, Laminar+SPS viscosity model and the value of $h / d_{p}=1.7$ hereafter for all numerical tests. The value of $h / d_{p}=1.7$ is also employed in [61] (see Section 4.4), who also stated that values ranging from 2 to 2.5 reduce the wave decay for larger numerical domains. However, our results of $h / d_{p}=2.1$ and 2.4 tend to be worse. This is a result of the larger particle sizes for our simulation compared with [61].

\subsection{Numerical conditions}

The numerical setup, including the particle distance $\left(d_{p}\right)$ and the total number of particles $\left(N_{p}\right)$, is reported in Table 2. In order to study and validate the convergence, the initial distance between the SPH particles in the computational model $\left(d_{p} / D\right)$ was set to $4.0 \times 10^{-3}, 2.0 \times 10^{-3}$ and $1.3 \times 10^{-3}$, where $D$ is the diameter of the FloWave basin. In the case of fine particles $\left(d_{p} / D\right.$ $=1.3 \times 10^{-3}$ ), a water depth to particle distance ratio, $h / d_{p}$ of 67 results in about 39 million particles $\left(N_{p}\right)$ for the circular wave basin, FloWave. The coefficient of smoothing length used in this model is 1.0. The variable time step was controlled by the Courant-Friedrichs-Lewy (CFL) condition under explicit time integration schemes. The total runtime was approximately $125 \mathrm{~h}$ for the total number of particles of about 39 million, $16 \mathrm{~h}$ for the middle particle size, $1 \mathrm{~h}$ for the coarse particle size for one million using a graphic processing unit (GPU). In this study, the initial water depth of $2.02 \mathrm{~m}$ for the fine particle case in the numerical tank was a little different from that in the experimental condition in FloWave because the initial particle distance could not divide the desired depth 
of $2.0 \mathrm{~m}$. From our experience, it was confirmed that the numerical results remain unaffected by the small difference of the initial water depth.

\section{Results and Discussion}

\subsection{Simulated Wave Fields}

A snapshot of the short-crested wave propagation from the negative side to the positive side along the $y$-axis as shown in Fig. 7 , at time $\mathrm{t}=30 \mathrm{~s}$ is presented in Fig. 12 for Cases $1 \sim 4$, showing the different degrees of spreading. Further assessing all of the time periods of the simulation, it was evident that, in all cases, wave fields were clearly generated and absorbed well, and this was visually similar to the waves generated at the experimental facility.

Figures 13 and 14 show spatial maps of the elevation of the water surface at time $t=30 \mathrm{~s}$ for all eight conditions, with sea state references shown in the top left corner of each subplot, which correspond to those defined in Table 1. These provide results for a range of sea states including both multi-directional and uni-directional waves, with high and low steepness values. The change in the wave fields is clearly apparent when varying with the spreading parameter $(s)$ : when $s=$ Inf., long-crested wave trains are visible along the $y$-axis, and conversely; when $s=25,10$ or 5 , short-crested wave fields occur in the circular wave basin. The wave trains in Fig. 13c $(s=25)$ resemble the long-crested waves in Fig. 13d ( $s=$ Inf.) waves, but the wave fields increasingly become short-crested with a decrease in $s$, with the most short-crested shown in Fig. 13a $(s=5)$. Moreover, the difference in wave steepness between Cases $1 \sim 4$ and Cases $5 \sim 8$ is apparent from the color contoured spatial variation; the wave crests in Cases $1 \sim 4$ are large and wavelengths are relatively small, whereas the opposite is observed in Cases $5 \sim 8$.

Furthermore, as illustrated in Figs. $15 \sim 20$, the numerical model simulated not only the water surface elevation but also the velocity, pressure fields in the FloWave basin. Those figures (Fig. $15 \sim$ Fig. 22) were described at a vertical section with a depth clearance of $d_{p}$. The velocity field at the $Y Z$-plane $(x=0)$ 
for Cases $1 \sim 4$ at time $t=30 \mathrm{~s}$ (shown as the central black dashed line in Figs. 13 and 14) are displayed in Fig. 15, as well as in Fig. 16 for Cases $5 \sim 8$. The black arrows indicate the vector of the velocity components at the $Y Z$-plane. Although wave fields (Figs. 13 and 14) become increasingly directionally spread as the spreading parameter $(s)$ decreases, similar velocity fields are evident for all spreading values in Figs. 15 and 16. This is further evident when assessing velocity fields and vectors close to the basin centre $(x, y=0)$, as shown in Figs. 17 and 18. This similarity is due to a combination of the dominant/mean wave propagation direction ( $y$-axis) being the same for all spreading conditions, and the fact that the same frequency-dependent phases (where at $x=0$ and $y=0$ ) are used. Figures $19 \sim 22$ show the pressure field at $Y Z$-plane $(x=0)$ for all wave conditions at time $t=30 \mathrm{~s}$, with stable pressure for all sea states. These results demonstrate the advantages of this numerical model, as both are difficult to obtain via experimental work, particularly over large areas. However, the velocity disturbance seems to be generated at the vertical sections with the depth clearance $d_{p}$, as shown in Figs. 17 and 18. This might be caused by both the particle density with particle numbers and directionally spread wave propagation in 3D multidimensional wave field in Figs. 13 and 14. The small gap between particles beneath the free surface is also found in Figs. 17 and 18. Thus, the finer spatial resolution discussed in Section 4.2.2 should be necessary for capturing more accurate velocity fields, and particle distribution to fulfil continuity at the free surface.

\subsection{Comparison to Measurements}

\subsubsection{Time-domain}

The time-series data for the measured and modelled (fine-resolution) surface elevations are shown in Fig. 23 for Cases $1 \sim 8$ in Table 1 . Generally, the model results compare favourably with the experimental data, and the wave magnitudes and the phases are reproduced in acceptable level.

Figure 24 shows a comparison between the numerical and experimental models of the water surface elevations recorded at WG4, at the centre of the basin 
$(x, y=0)$, for Cases $1 \sim 4$. Figure 25 shows the measured time-series of water elevations for Cases $5 \sim 8$ recorded at WG4. The case numbers defined in Table 1 are shown in the left corner of each row. From Fig. 24 and 25, it is apparent that the time-series data of surface elevations measured at WG 4 are similar for all sea states, including both short- and long-crested waves, as the frequency-dependent phases (defined at $x=0$ and $y=0$ ) are the same for all spreading values. These results demonstrate that all sea states were reproduced using the single summation method that was explained in Section 2.3.

In terms of different particle resolutions, it is evident that the very-coarseresolution $\left(d p / D=4.0 \times 10^{-3}\right)$ results differ more significantly from the experimental measurements than the finer-resolution results; the phases for the coarse-resolution model are considerably different from the experimental data, and their peaks are also reduced. In comparison, the results obtained with the fine particle resolution $\left(d p / D=1.3 \times 10^{-3}\right)$ show practical agreement with the experimental data. The phase and wave height crests are close to the experimental results, although there is a tendency of underestimate. This is because it is difficult to accurately capture higher-frequency waves, especially in cases with larger wave steepness (Cases $1 \sim 4$ shown in Fig. 24), even though the fine particle results captured the elevation tendencies.

\subsubsection{Statistical Analysis}

Figure 26 shows comparisons of significant wave height $\left(H_{s}\right)$ and mean wave periods $\left(T_{m}\right)$ for the experimental and numerical results obtained for fine particles. These results were calculated using time-series data for surface elevation defined by a down-crossing method at each gauge shown in Fig. 5 from time $t=20$ to $64 \mathrm{~s}$. The first $20 \mathrm{~s}$ were discarded to enable the high-frequency wave components to arrive at the measurement location, thus enabling a more accurate comparison of the sea state generation performance.

There is a tendency for the model to underestimate in wave height for Cases $1 \sim 4$, with large amplitudes $H_{s}=0.15 \mathrm{~m}$; however, the values for Cases $5 \sim$ 8 showed an acceptable agreement with the experimental data. The significant 
wave height percentage errors, defined as $\left(H_{s-\mathrm{SPH}} / H_{s \text {-EXP }}-1\right) \times 100$, ranged from $-10 \%(s=5)$ to $-16 \%$ ( $s=$ Inf.) for Cases $1 \sim 4$, and from $-8 \%(s=5)$ to $+1 \%$ ( $s=$ Inf.) for Cases 5 to 8 . For all of the cases, the measured $T_{m}$ values were higher in the model in the model than those obtained experimentally because of the inability of the model to fully capture the variance associated with the highest-frequency wave components.

The difference observed in the results for the low and high steepness cases is explained by the particle size relative to the wavelengths. For the higher period sea states (Cases $5 \sim 8$ ), the particles are very small relative to the majority of the wavelengths; hence, the model could capture the sea-surface deformation relatively accurately. On the other hand, for Cases $1 \sim 4$, which have lower peak periods, the apparent particle size is larger relative to the wavelength and could not describe the wave motion as accurately. This is analogous to spatial averaging which results in the peaks of the higher-frequency waves not being properly captured, thereby reducing the significant wave heights and increasing the mean observed wave periods. It should be also noted that the number of particles sitting in a wave height $\left(H / d_{p}\right)$ is lower than recommended value $\left(H / d_{p}>10\right)$ proposed in [61]. This lack of particles against wave height becomes remarkable in high frequency components, as the waves have small wave heights, which can be estimated from Fig. 4.

\subsection{Convergence}

In order to analyse the model convergence, the $r^{2}$ (coefficient of determination) values for all cases and combinations of significant wave heights at WG4 ( $x$, $y=0)$ and the number of particles for target significant wave height $\left(H_{s_{-}}\right.$target $)$ are shown in Figs. 27 and Fig. 28, respectively. Similar to the statistical comparisons, only the final $44 \mathrm{~s}$ of data (from time $t=20$ to $64 \mathrm{~s}$ ) were used for comparison.

Based on Fig. 27, the $r^{2}$-values increased with the reduction in $d_{p}$. Focusing on coarse particles for lower-steepness sea states (Sea States 5-1, 6-1, 7-1 and $8-1)$, the $r^{2}$-values were approximately 0.5 at all gauges; however, the $r^{2}$-value 
for fine particles was over 0.7. For the sea states with larger wave steepness (Cases $1 \sim 4$ ), the $r^{2}$-values were notably, if not significantly, lower (even at a finer resolution), although the values increased with a reduction in $d_{p}$. These results re-iterate the finding that lower-steepness states were better reproduced in the model compared with higher-steepness cases.

This finding is further shown in the significant wave height errors corresponding to number of particles in $H_{s}$-target, shown in Fig. 28. For lower-steepness cases (Cases 5-8), the associated significant wave heights with coarse particles are clearly underestimated; however, the errors notably reduce with the increase of particle number on $H_{s}$ target. For fine particle cases (i.e. 2.5 particles on the $H_{s_{-}}$target), the calculated significant wave heights are generally equivalent to the experimentally measured significant wave heights. In contrast, for larger-steepness cases (Cases 1-4), the significant wave heights with fine particles are still underestimated, although not considerably, even though the associated phases are practically reproduced for both lower- and larger-steepness sea states (see Figs. 24 and 25). All error percentages for the associated significant wave heights for the numerical results and experimental data are shown in Table 3.

The simulated wave heights for the lower-steepness cases are close to those obtained experimentally. Noting that the high frequency components are not fully reproduced (Fig. 23b), in comparison, the lower-frequency components are effectively replicated (as can be visually seen). As high wave frequencies tend not to significantly affect OEC response and loads, and the low-frequency components are well produced, it is suggested that the fine particle model will be suitable for the numerical exploration of OEC's responses in short-crested waves. It should be noted that longer runs, or the averaging of many shorter (e.g. 64s) realisations, are required for robust statistical assessment of OEC performance in specific sea states. The latter is currently possible with the presented model. 


\section{Conclusions and Future Works}

Numerical wave basins have been strongly required as a powerful tool to investigate fluid-structure interaction (FSI) problems in coastal and ocean engineering fields. In this study, for the first time we created, and validated, the generation of short-crested wave conditions using a full 3D NSE based numerical model of a circular wave basin using a particle-based method. The numerical tank model was based on the FloWave facility at the University of Edinburgh: the geometry, 168 hinged-flap wave makers mounted around entire circumference, enables the generation of multi-directional waves. Multi-directional waves were generated using the single-summation method on the basis of the PiersonMoskowitz spectrum and cosine-2s spreading function with differing values of steepness and directional spreading, and simulated surface elevations were compared to experimentally-obtained data. Based on our findings, the main conclusions and recommended areas for future work are as follows:

- The numerical tank model reproduced long- and short-crested waves using the dynamic boundary particles of 168 hinged-flap-type wavemakers. The modelled time histories water elevations showed an overall practical agreement with the experimental data. The lower- frequencies and steepness model, Cases $5 \sim 8$, were better reproduced. The $r^{2}$-values between the experimental and numerical water surface elevations were greater than 0.7 , and the significant wave height error ranged from $-8 \%$ to $+1 \%$.

- For Cases $1 \sim 4$, which have high frequencies and steepness, the percentage error between the numerical and experimental data for significant wave height when using fine particles ranged from $-10 \%$ to $-16 \%$ (i.e. underestimate). This is attributed to the underestimate of high-frequency waves. It is concluded that a greater number of particles is required to accurately capture the smaller higher frequency disturbances.

- It is suggested, however, that as the lower-frequency components are well produced for both low and high steepness cases that the model is already 
${ }_{446}$ suitable for the majority of FSI problems in both uni- and multi-directional

${ }_{447} \quad$ wave fields. These findings can form the basis for a wave-current simulation which future work aims to deal with. 


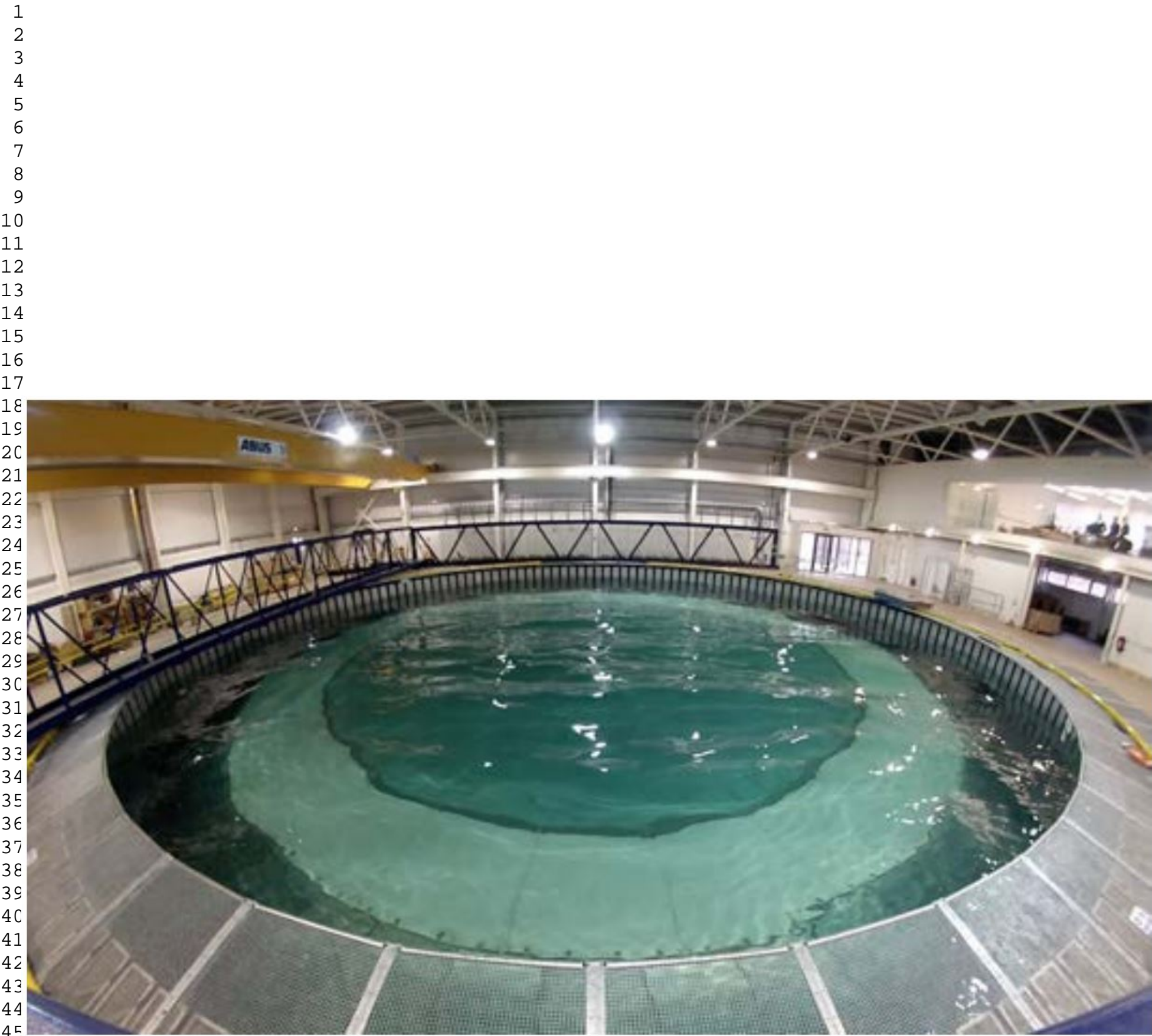

46

47 


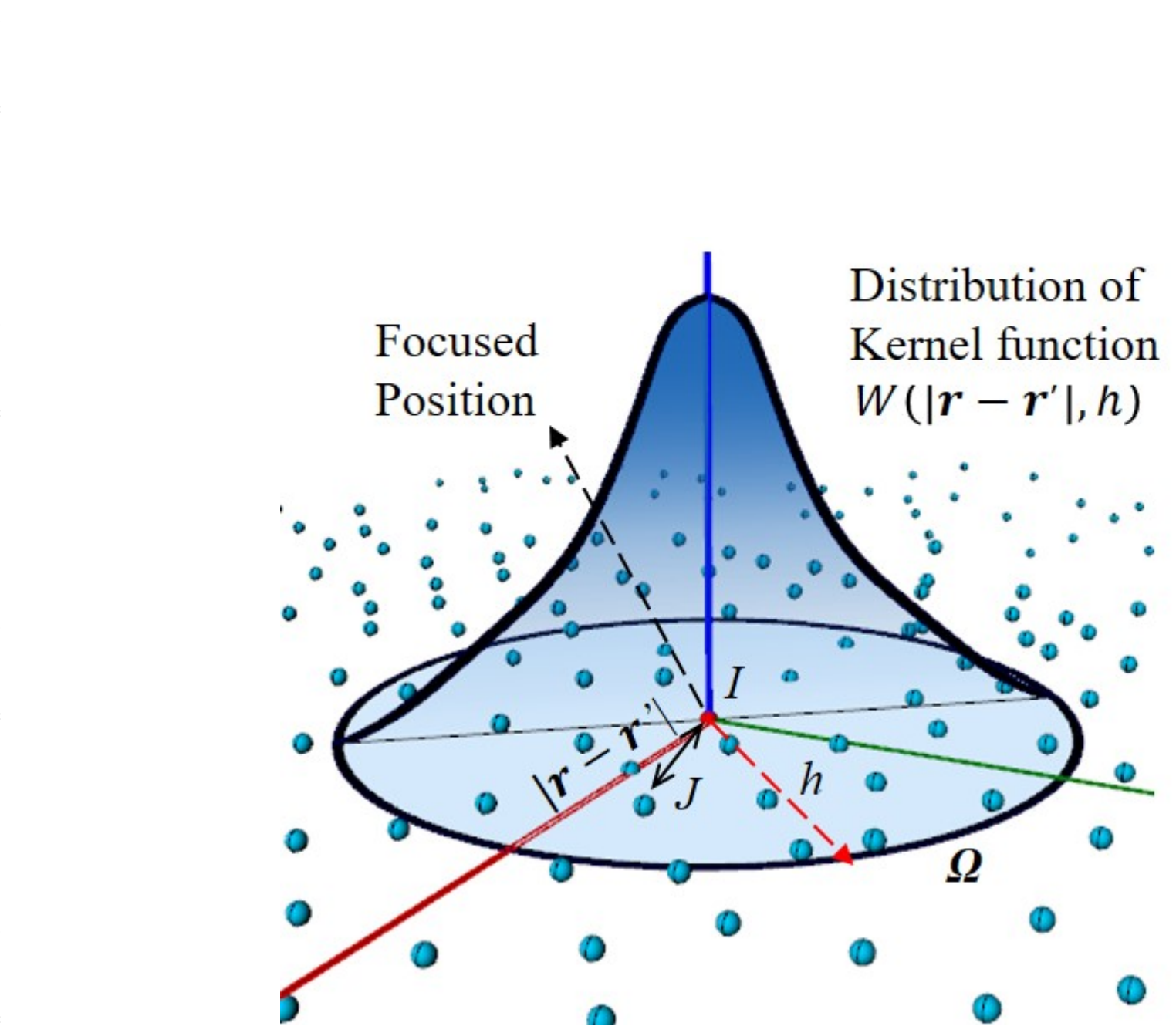

Figure 2: The fundamental principle of the SPH method represented by distribution of a Kernel function with a referenced distance, $h$. 


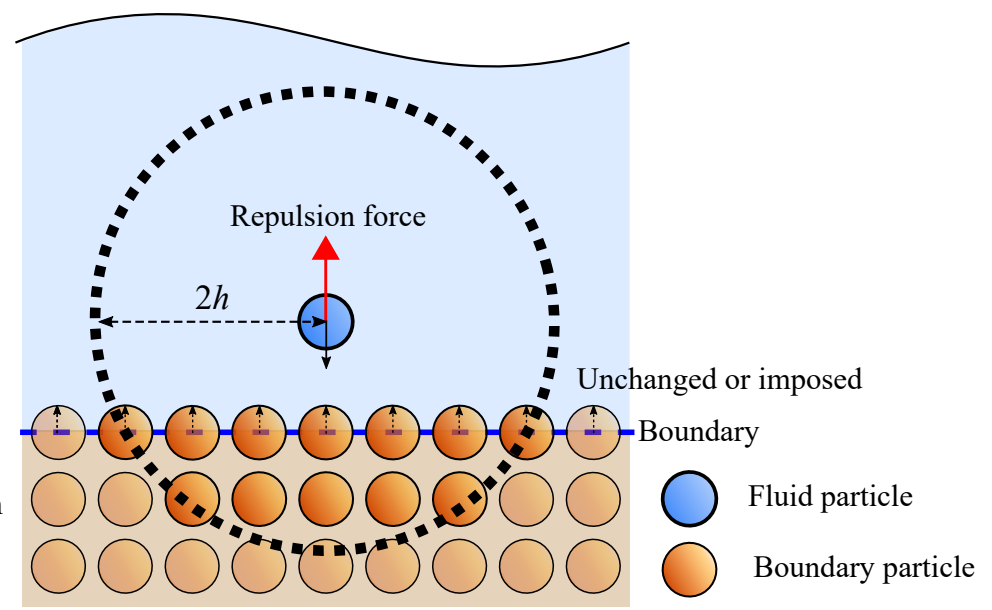

Figure 3: Sketch of the repulsion force in dynamic boundary condition

Table 1: Target wave specification for the eight cases generated in FloWave.

\begin{tabular}{c|c|c|c|c|c}
\hline Case & $H_{m 0}[\mathrm{~m}]$ & $T_{p}[\mathrm{~s}]$ & $s$ & Sea states & Comp. time $[\mathrm{s}]$ \\
\hline 1 & 0.15 & 1.5 & 5 & Short-crested & 64 \\
2 & 0.15 & 1.5 & 10 & Short-crested & 64 \\
3 & 0.15 & 1.5 & 25 & Short-crested & 64 \\
4 & 0.15 & 1.5 & Inf. & Long-crested & 64 \\
\hline 5 & 0.075 & 3.0 & 5 & Short-crested & 64 \\
6 & 0.075 & 3.0 & 10 & Short-crested & 64 \\
7 & 0.075 & 3.0 & 25 & Short-crested & 64 \\
8 & 0.075 & 3.0 & Inf. & Long-crested & 64 \\
\hline
\end{tabular}



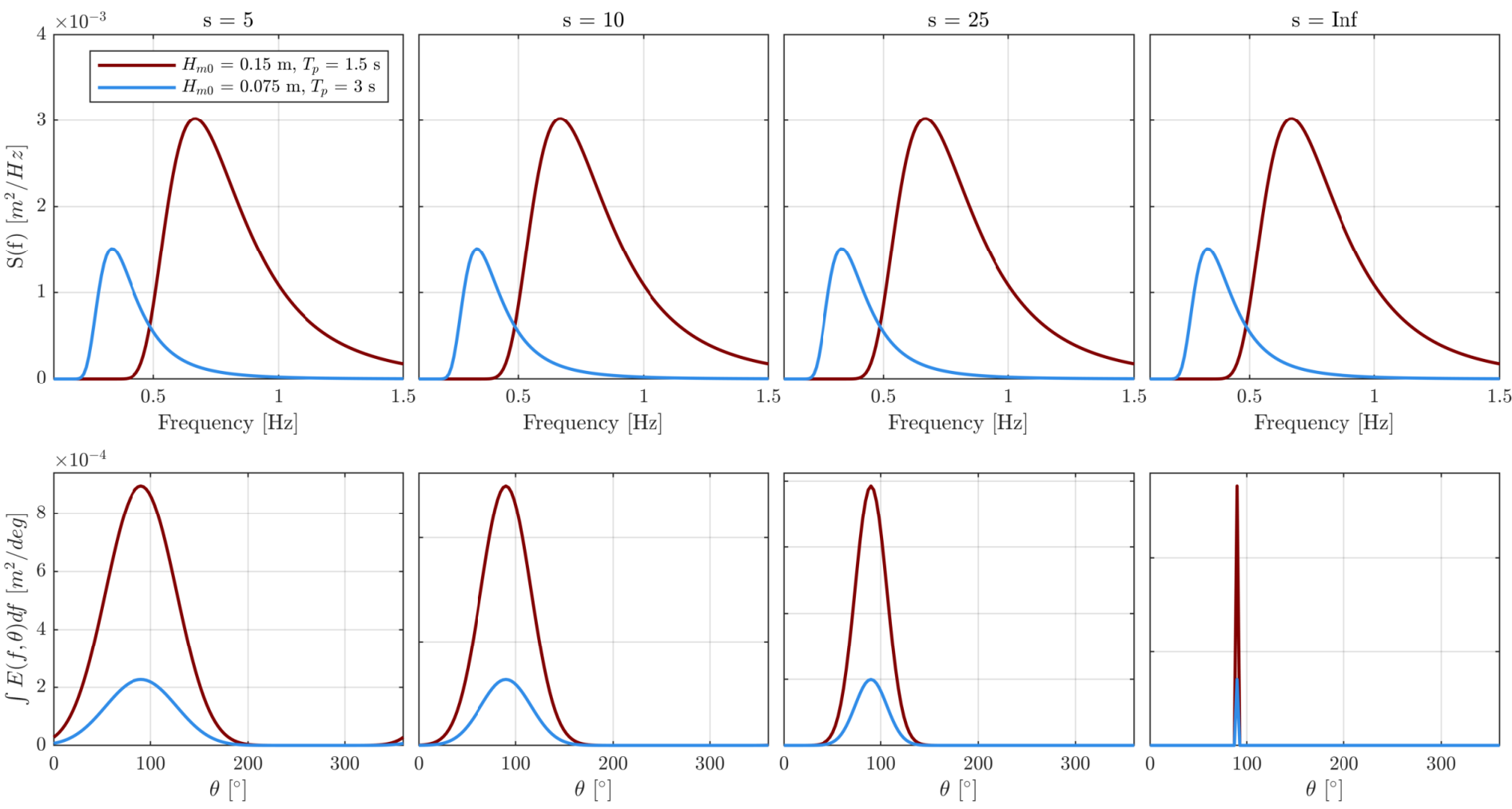

5

51

5

53

54

55

56 


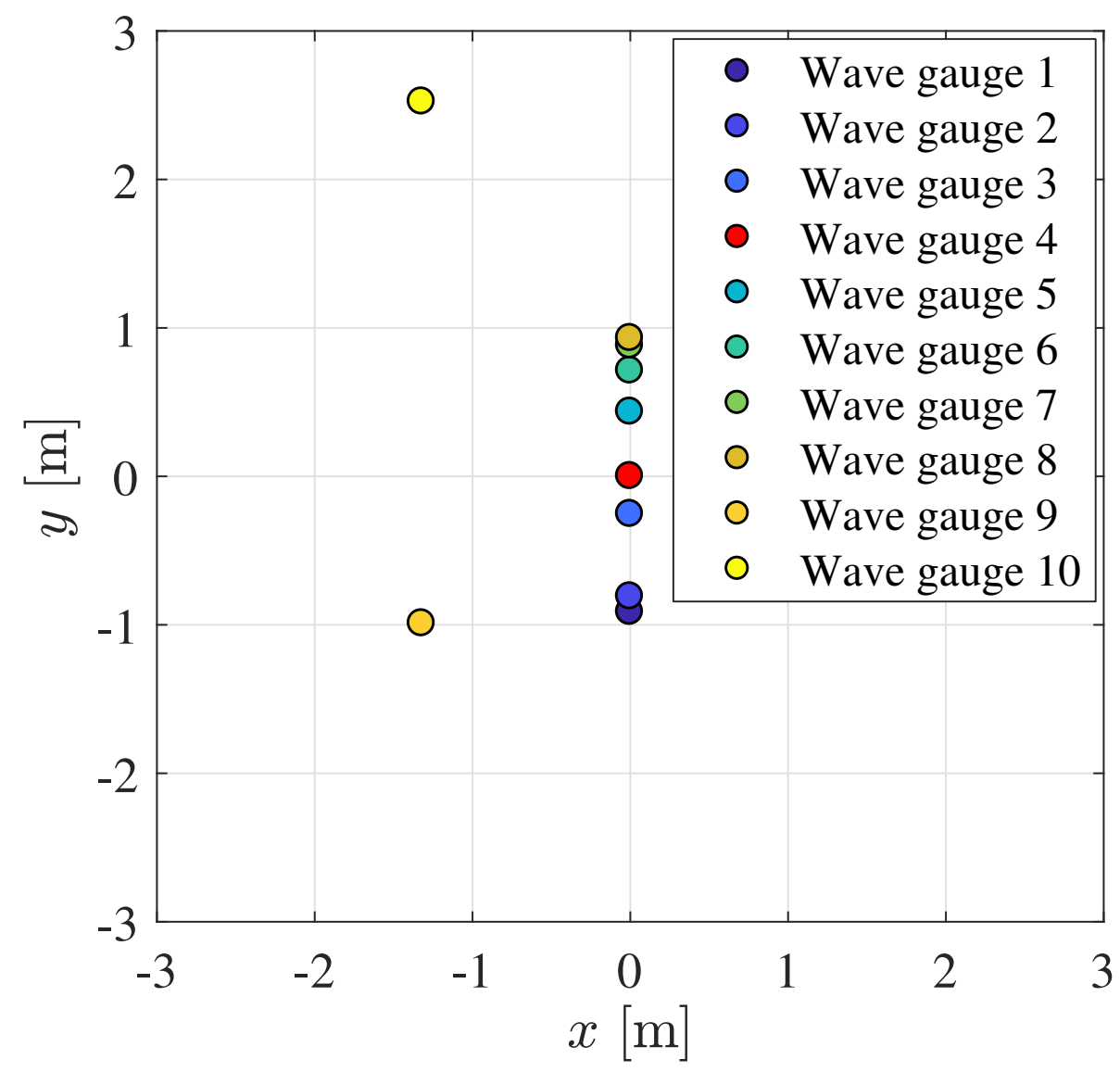

Figure 5: WG array layout close to the centre of FloWave shown in Fig. 7. The red circle (WG4) represents the centre of the FloWave tank $(x, y=0)$. 

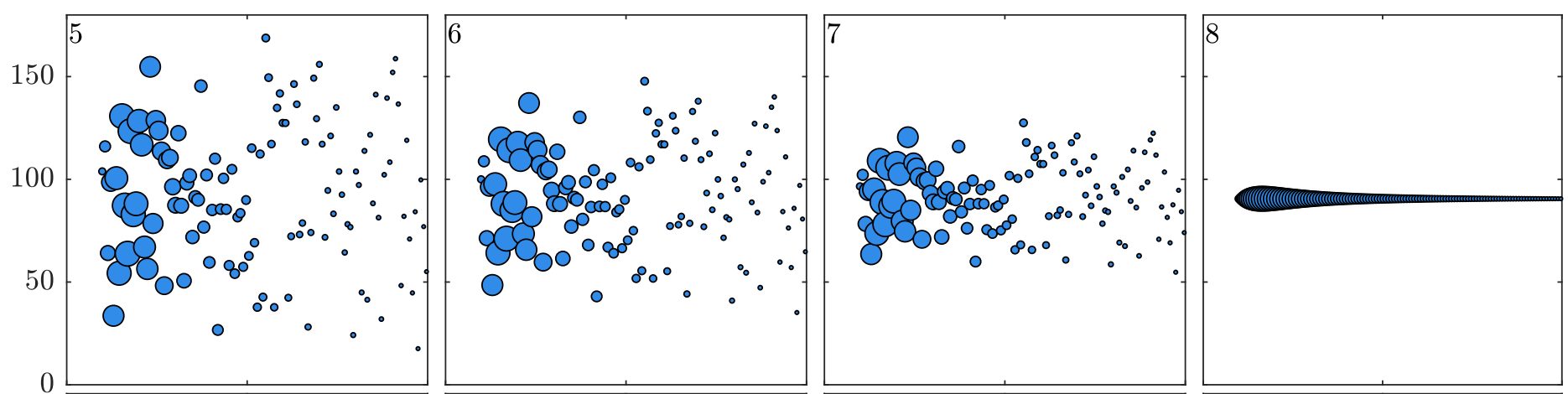

45Figure 6: Input fronts used for Cases $1 \sim 8$. The size of each marker is proportional to the amplitude used. Case numbers are shown in the top left 46 
58Figure 7: Schematic numerical configuration of the FloWave tank encircled by 168 hinged-flap-type wavemakers with a top view of its coordinate

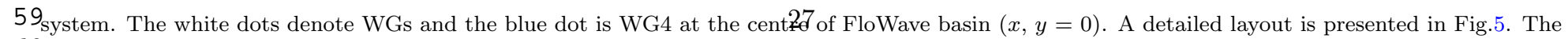
60 61 
10

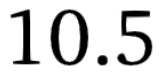

8.5 8 .

8. 5 \%o0000000000000000000000000

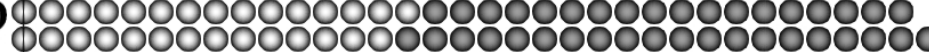

000000000000000000000000000000000



\$00000000000000000000000000000000000

000000000000000000000000000000000009

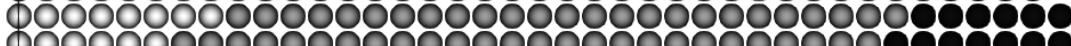

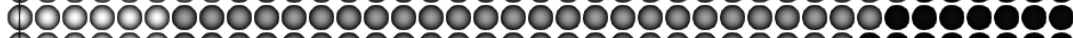

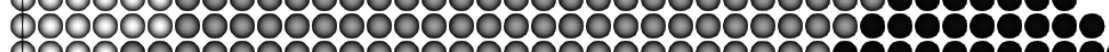

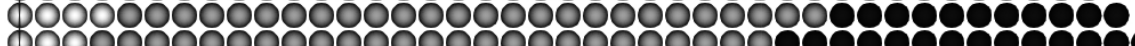

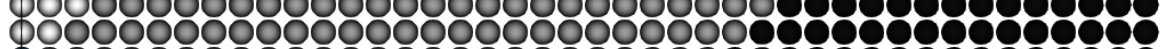



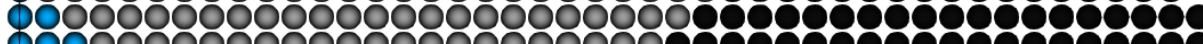

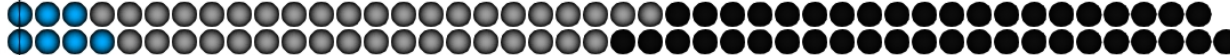

8,0000006

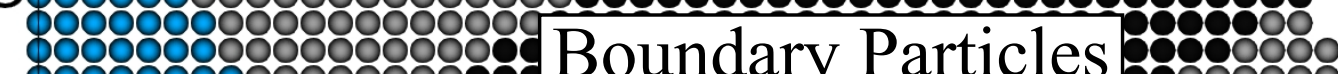

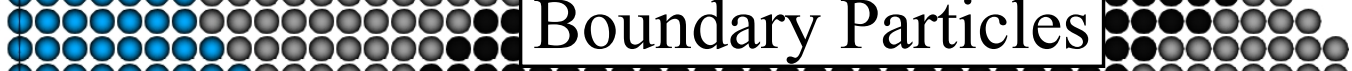

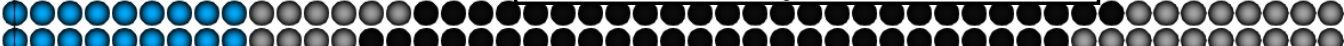

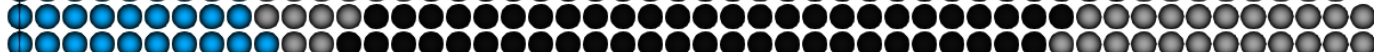

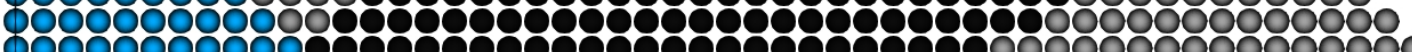


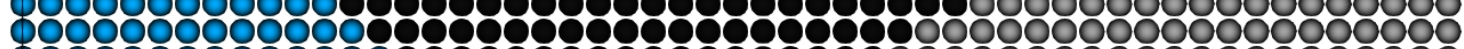

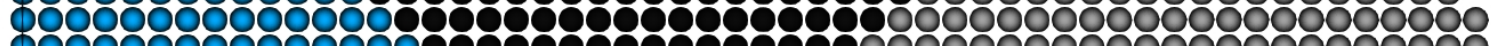


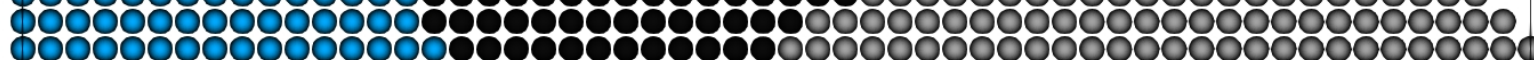

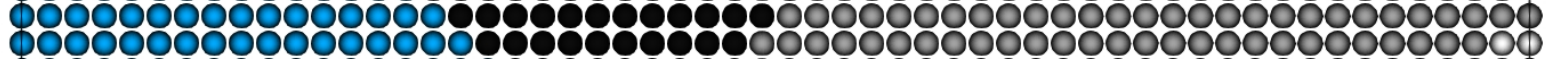

7 . 5 \%

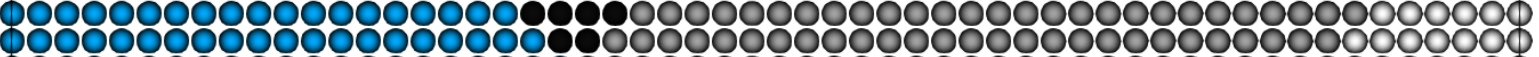

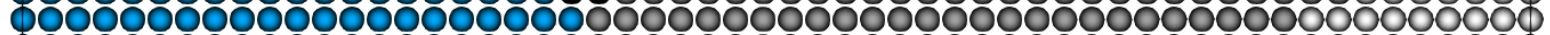

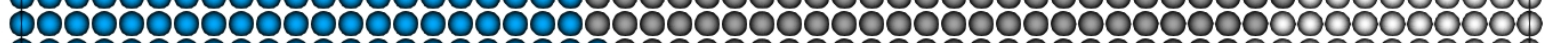
10000000000000000000000000000000000000000000000000 00000000000000000000000000000000000000000000000000000

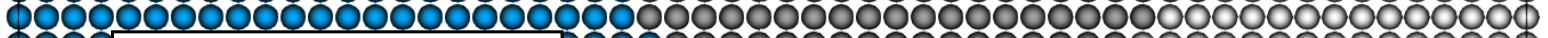
\&og Fluid Particles 3000000 ood 1 I I I I

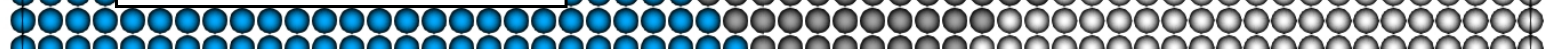

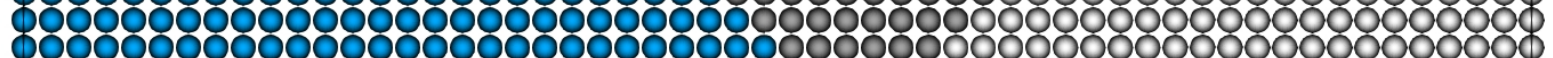

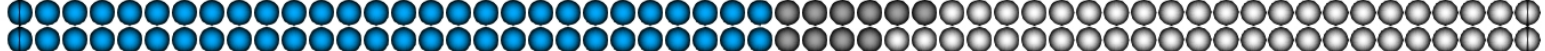

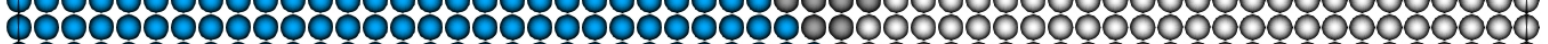
7 \%

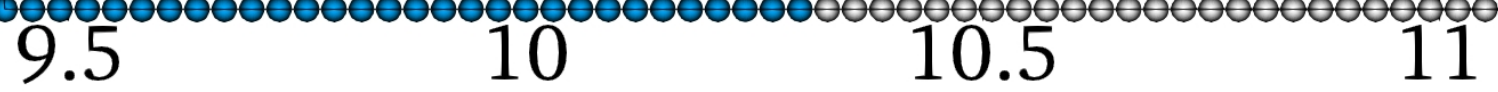

\section{$x[\mathfrak{M}]$}

Figure 8: Top view of particle distributions around the wave paddles. The blue dots represent fluid particles and the gray dots represent boundary

(1)




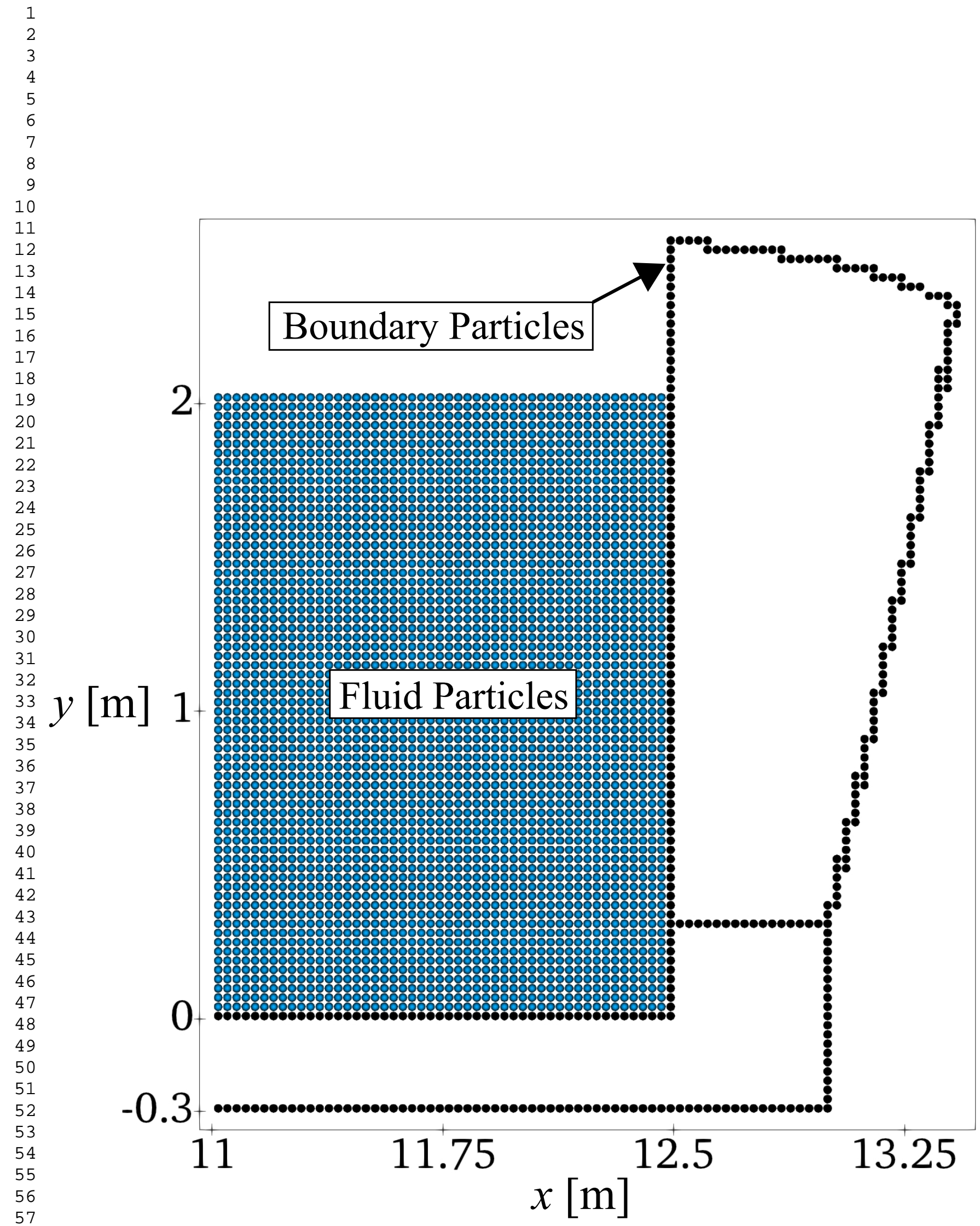

58

59Figure 9: Cross-sectional view of particle distributions around a wavemakex 9 The blue dots represent fluid particles with a water depth of $2 \mathrm{~m}$ and the

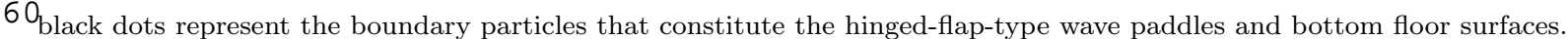

61 


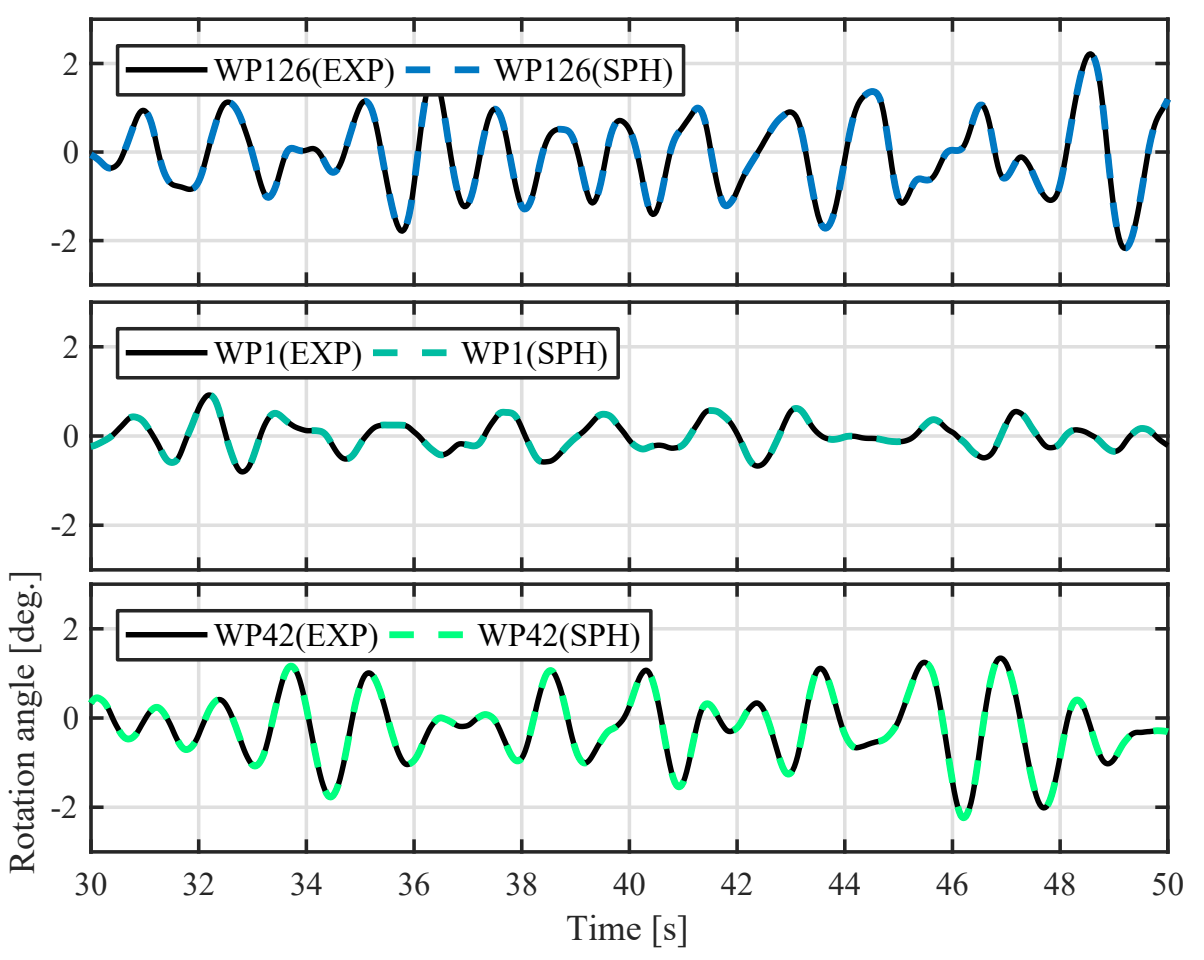

Figure 10: Example of time series of the recorded paddle angle rotation, which are imposed in the SPH model as the same boundary conditions in experiments for Case 1 presented in Table 1 . 

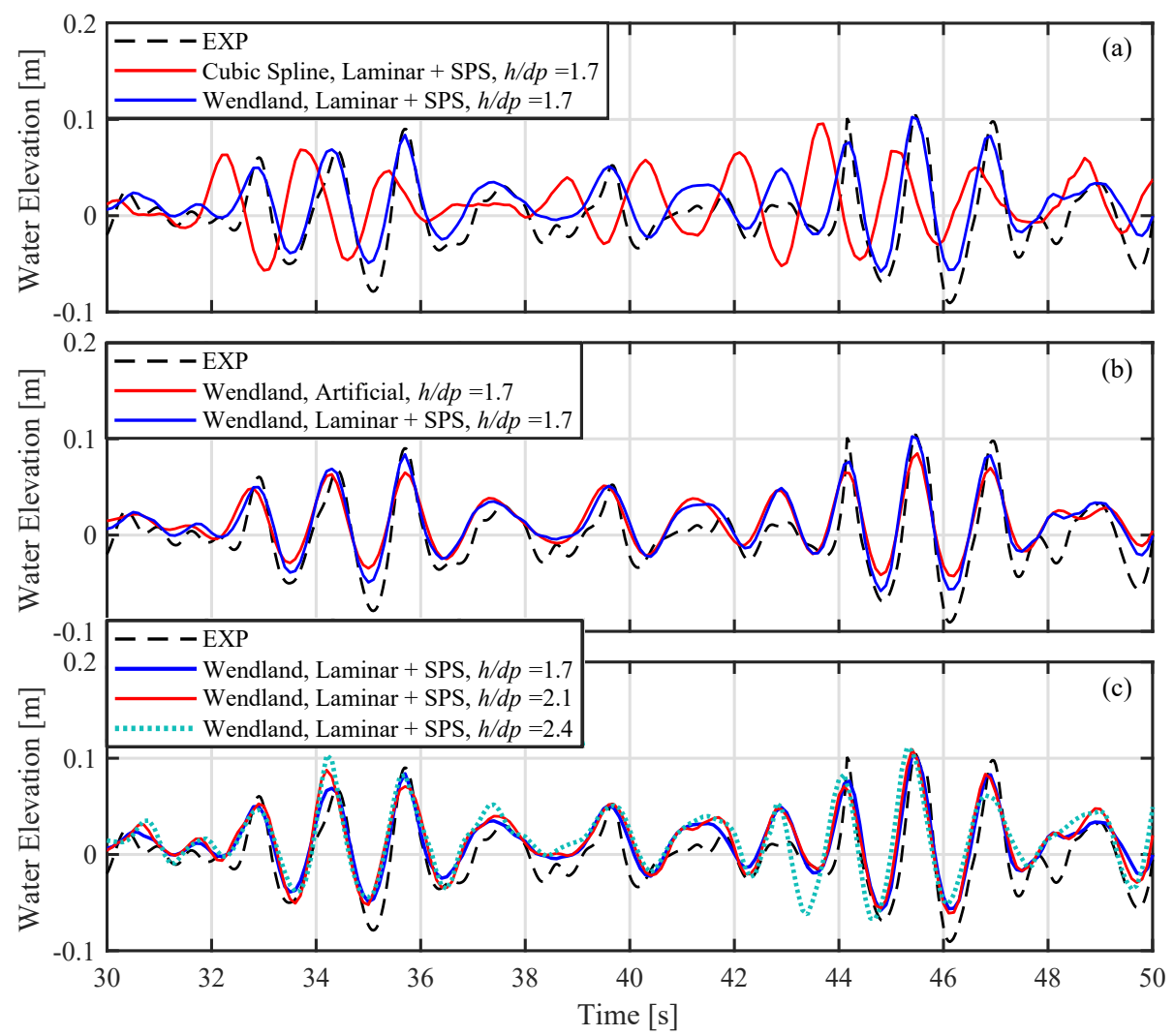

Figure 11: Examples of time series of water surface elevation for Case 4, with various numerical option; (a) Tow different kernel function used, (b) Showing the difference between two viscosity schemes and (c) Three different $h / d_{p}$ values are used. 
Table 2: Characteristics and runtimes for 24 cases with different resolutions.

\begin{tabular}{|c|c|c|c|}
\hline Sea states & $d p / D\left(\times 10^{-3}\right)$ & $h / d p$ & $N_{p}\left(\times 10^{6}\right)$ \\
\hline $1-1$ & 4.0 & 20 & 1.1 \\
\hline $1-2$ & 2.0 & 40 & 8.6 \\
\hline $1-3$ & 1.3 & 67 & 38.7 \\
\hline $2-1$ & 4.0 & 20 & 1.1 \\
\hline $2-2$ & 2.0 & 40 & 8.6 \\
\hline $2-3$ & 1.3 & 67 & 38.7 \\
\hline $3-1$ & 4.0 & 20 & 1.1 \\
\hline $3-2$ & 2.0 & 40 & 8.6 \\
\hline $3-3$ & 1.3 & 67 & 38.7 \\
\hline $4-1$ & 4.0 & 20 & 1.1 \\
\hline $4-2$ & 2.0 & 40 & 8.6 \\
\hline $4-3$ & 1.3 & 67 & 38.7 \\
\hline $5-1$ & 4.0 & 20 & 1.1 \\
\hline $5-2$ & 2.0 & 40 & 8.6 \\
\hline $5-3$ & 1.3 & 67 & 38.7 \\
\hline $6-1$ & 4.0 & 20 & 1.1 \\
\hline $6-2$ & 2.0 & 40 & 8.6 \\
\hline $6-3$ & 1.3 & 67 & 38.7 \\
\hline $7-1$ & 4.0 & 20 & 1.1 \\
\hline $7-2$ & 2.0 & 40 & 8.6 \\
\hline $7-3$ & 1.3 & 67 & 38.7 \\
\hline 8-1 & 4.0 & 20 & 1.1 \\
\hline $8-2$ & 2.0 & 40 & 8.6 \\
\hline $8-3$ & 1.3 & 67 & 38.7 \\
\hline
\end{tabular}

$d_{p}:$ the particle size.

$D$ : the diameter of the circular numerical tank, FloWave.

$h:$ the initial water depth in circular numerical tank, FloWave.

$N_{p}$ : the total number of particle. 


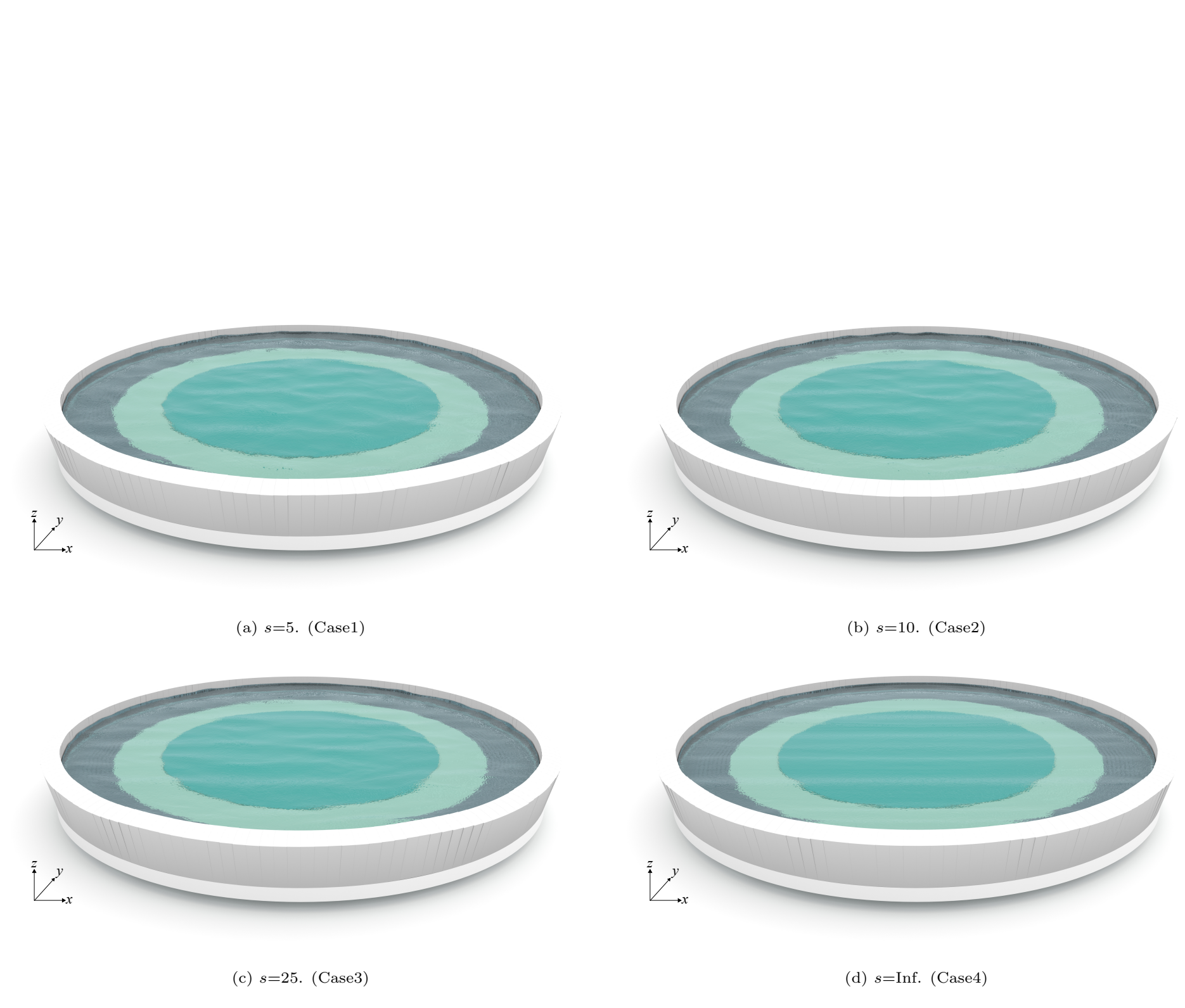

41

42Figure 12: Example snapshots of wave states under short-crested wave conditions for Cases $1 \sim 3$ where the spreading parameter $s=5$, 10 and 25 , as 43 well as a long-crested wave for Case 4 where $s=$ Inf. (the mean wave direction is from the negative side to the positive side along the $y$-axis). 44

45

46

47

48

49

50

51

52

53

54

55

56

57

58

59

60

61

62

63 


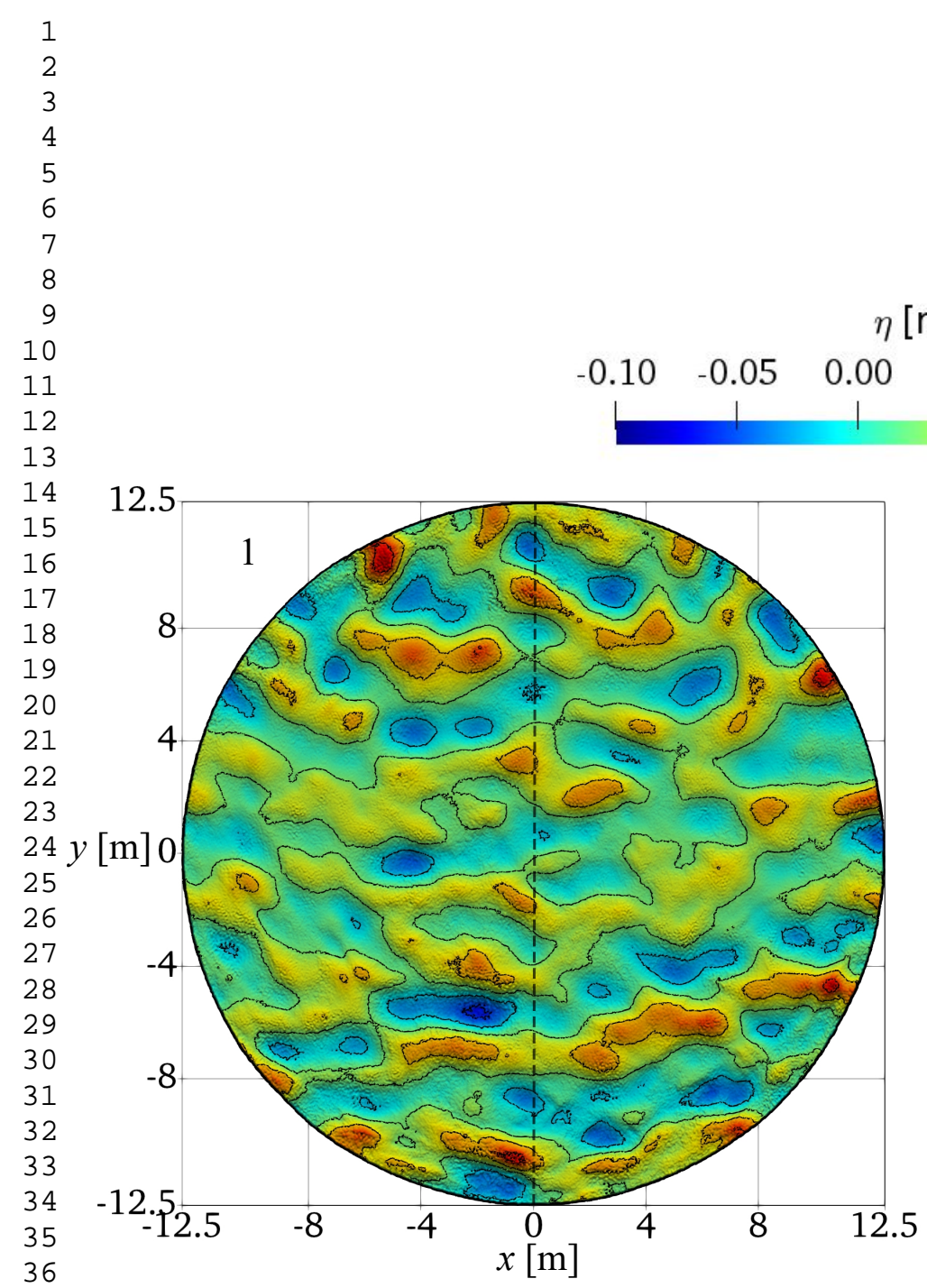

(a) $s=5$

38

39

40

41

42

43

44

45

46

47

48

59

51

52

53

54

55

56

57

58

59

60

61

62

63

64Figure 13: Spatial maps of water elevation in short-crested wave fields where (a) $s=5$, (b) $s=10$ and (c) $s=25$, as well as in a long-crested wave

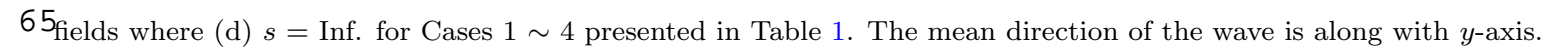

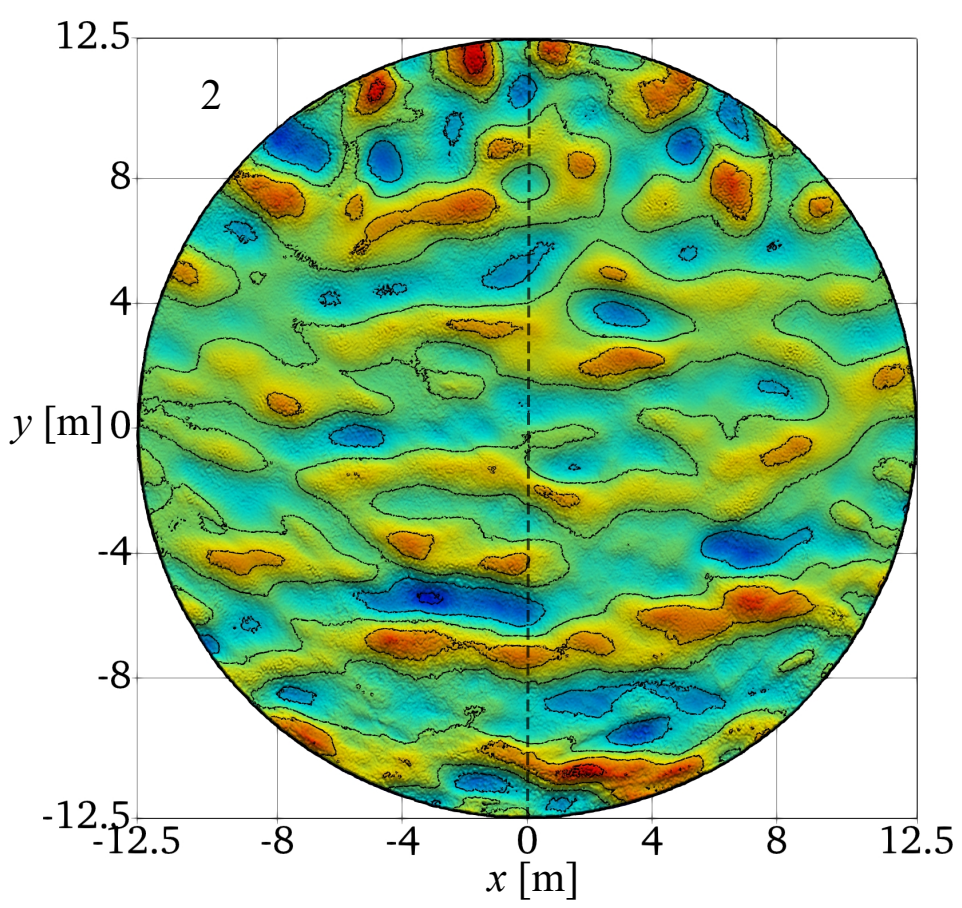

(b) $s=10$

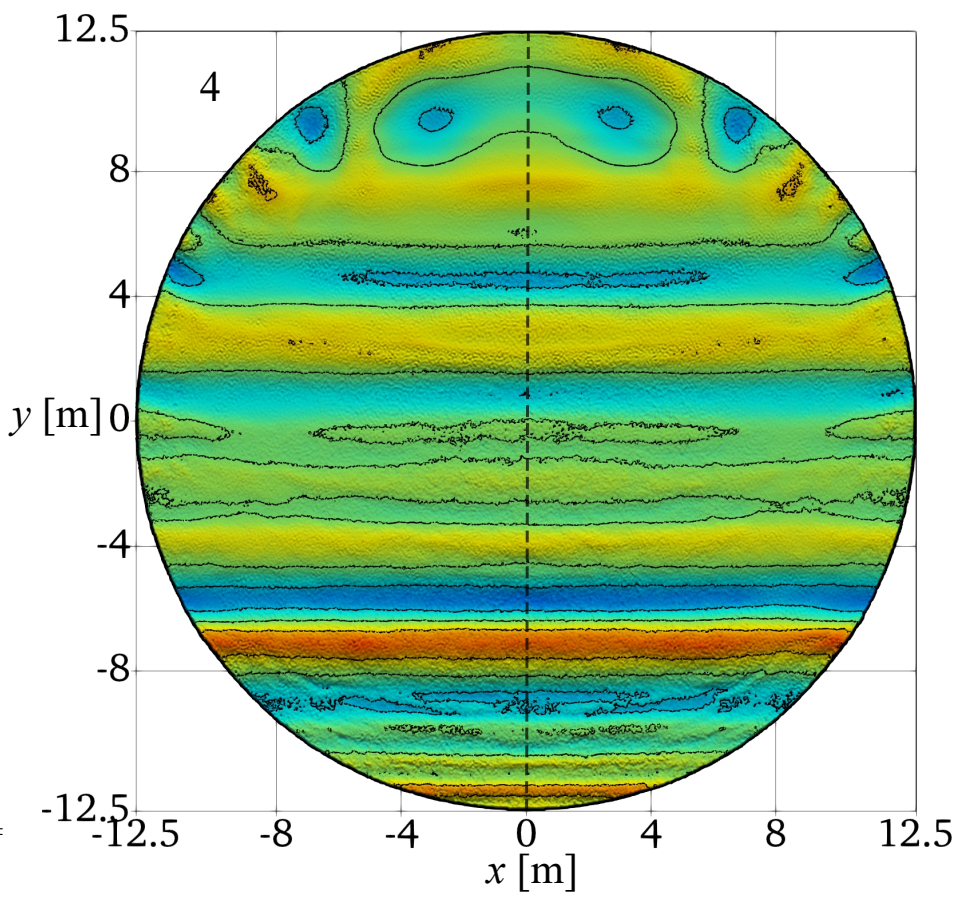

(d) $s=$ Inf.

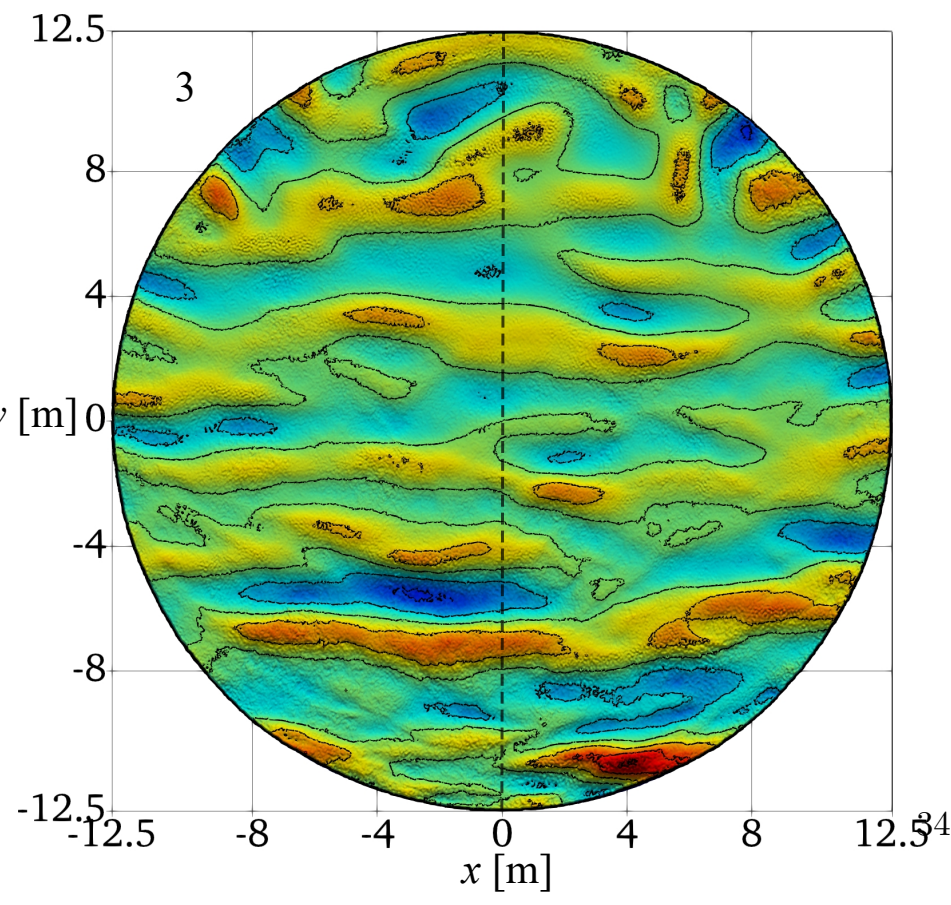

(c) $s=25$
$0.05 \quad 0.10 \quad 0.15$ 


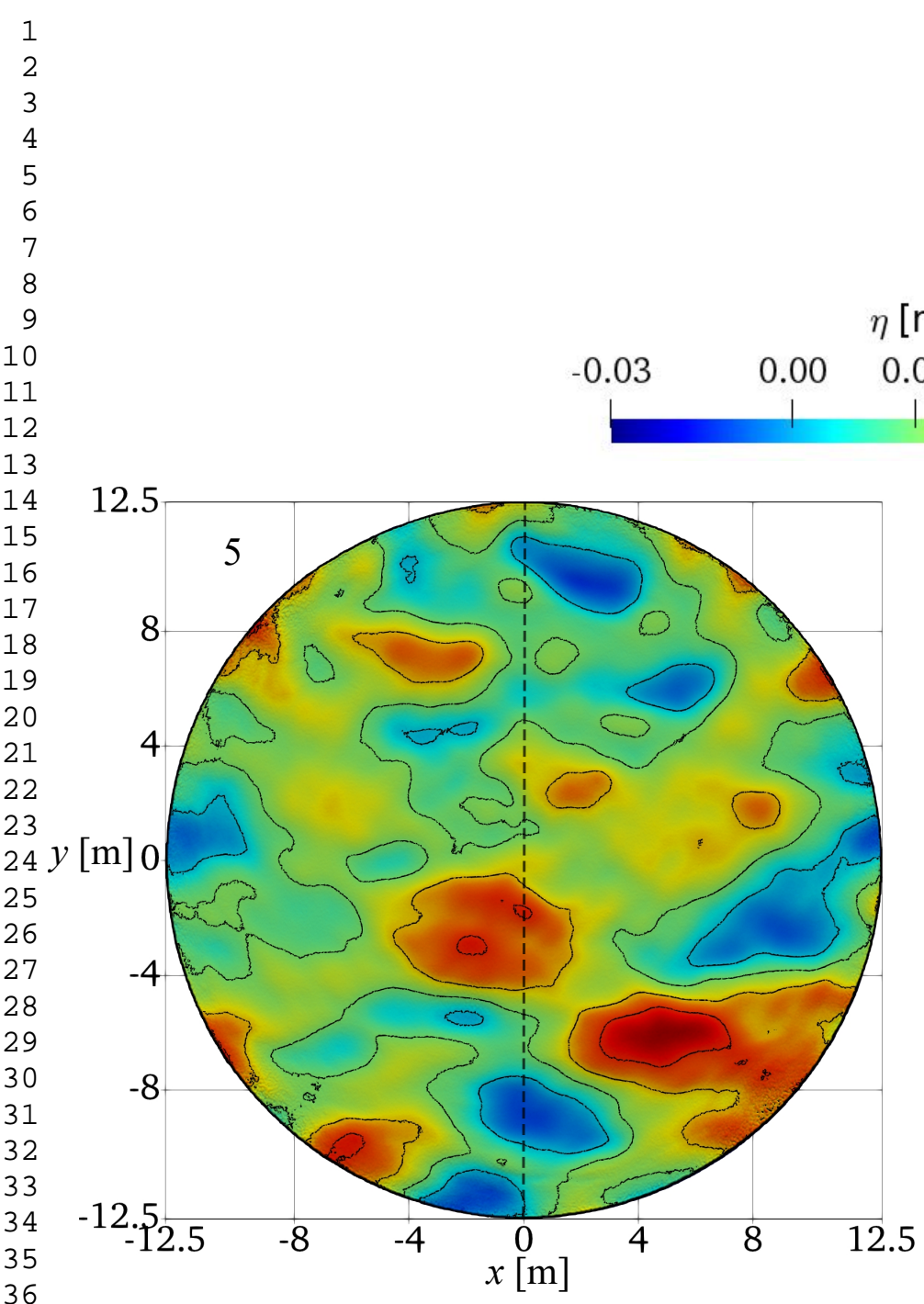

36

37

38

39

40

41

42

43

44

45

46

47

48

50

51

52

53

54

55

56

57

58

59

60

61

62

63

64Figure 14: Spatial maps of water elevation in short-crested wave fields where (a) $s=5$, (b) $s=10$ and (c) $s=25$, as well as in a long-crested wave 65field where (d) $s=$ Inf. for Cases $5 \sim 8$ presented in Table 1. The mean direction of the wave is along with $y$-axis.

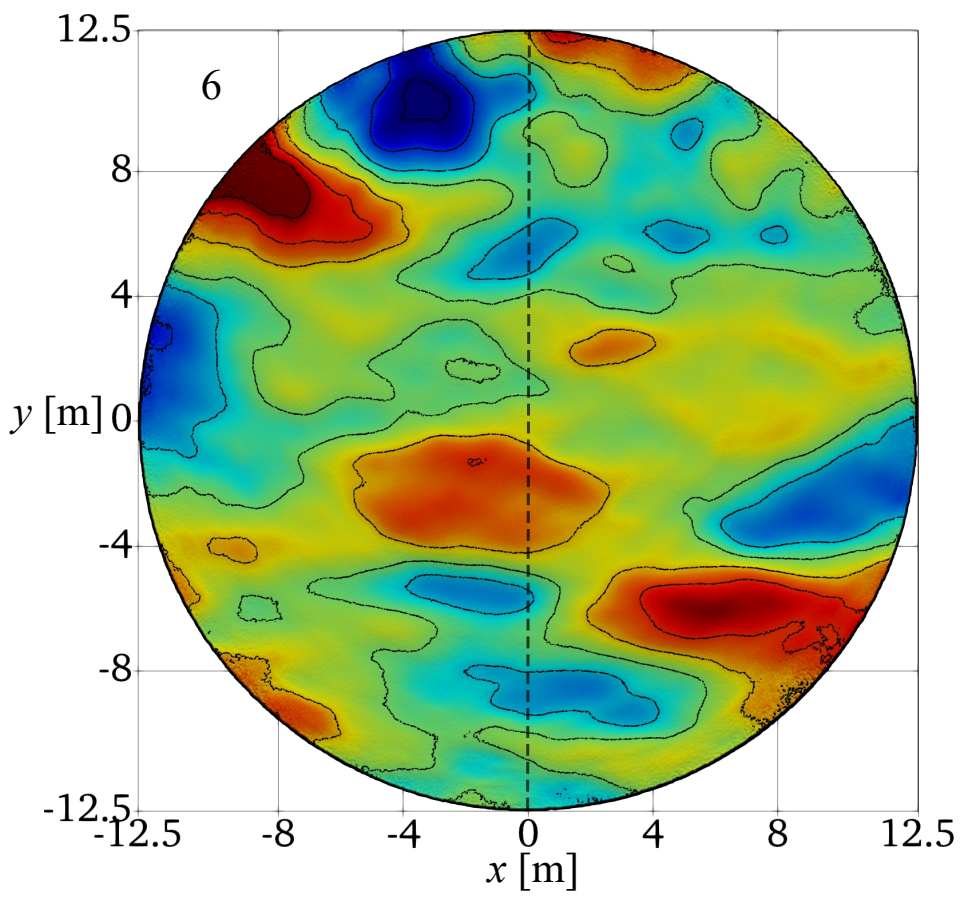

(a) $s=5$

(b) $s=10$

(d) $s=$ Inf.

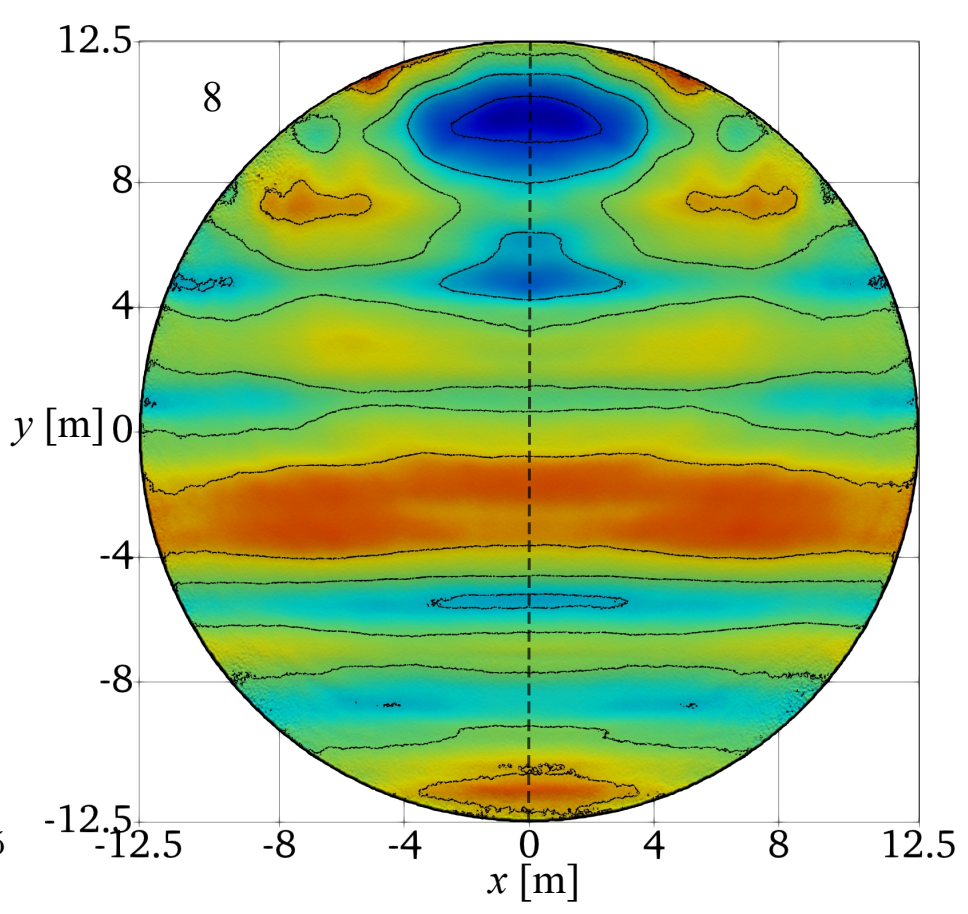

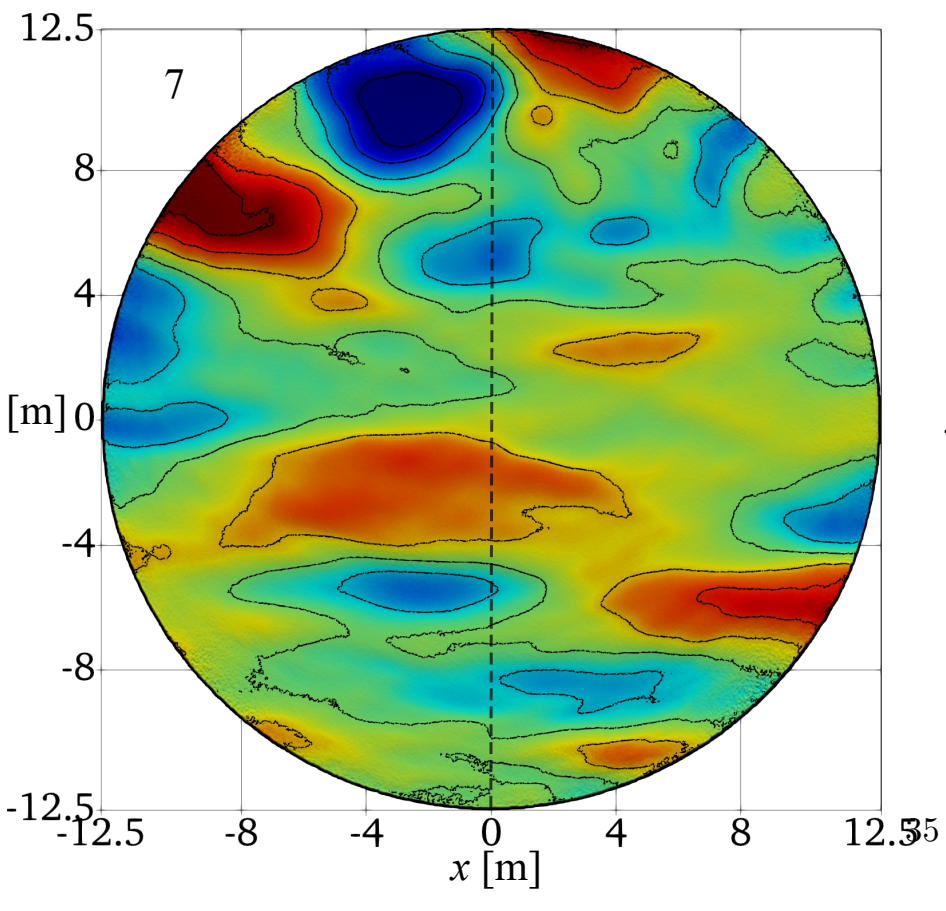

(c) $s=25$ 
49Figure 15: Snapshots of the velocity fields at the $Y Z$-plane with $x=0$, as indicated by the black dashed line in Fig. 13 , for Cases $1 \sim 4$ at $t=30 \mathrm{~s}$.
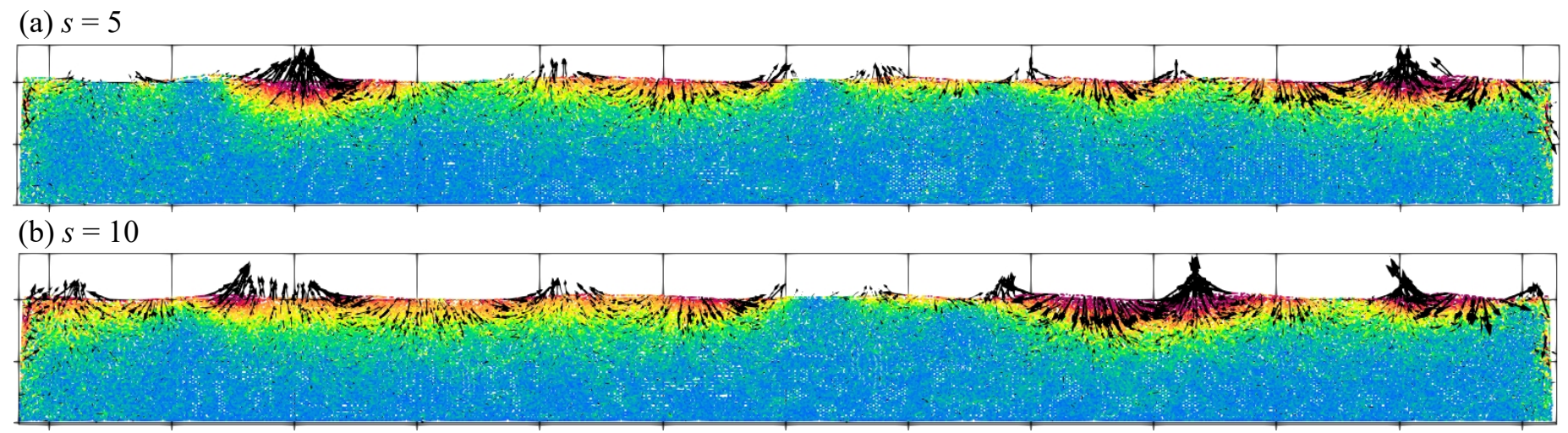

\section{(c) $s=25$}

(d) $s=$ Inf.

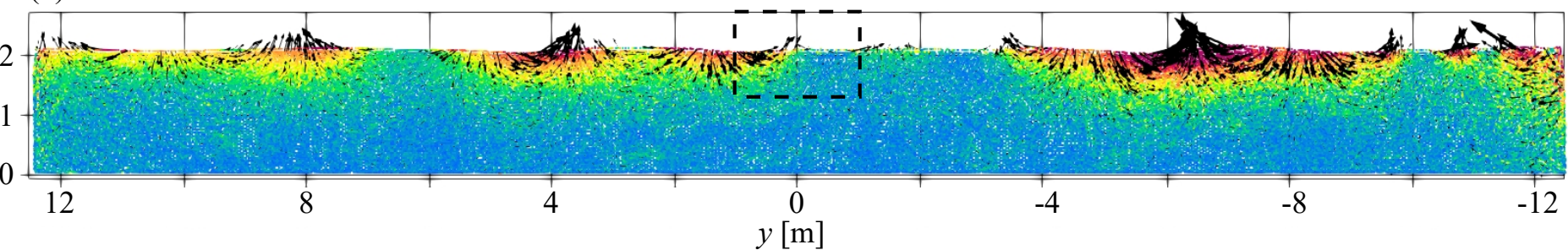


$49^{F i g u r e ~ 16: ~ S n a p s h o t s ~ o f ~ t h e ~ v e l o c i t y ~ f i e l d s ~ a t ~ t h e ~} Y Z$-plane where $x=0$, as indicated by the black dashed line in Fig. 14, for Cases $5 \sim 8$ at $t=30 \mathrm{~s}$. 50

(a) $s=5$

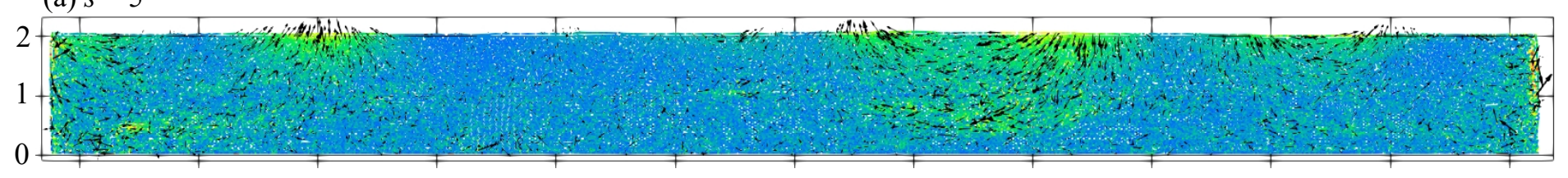

(b) $s=10$

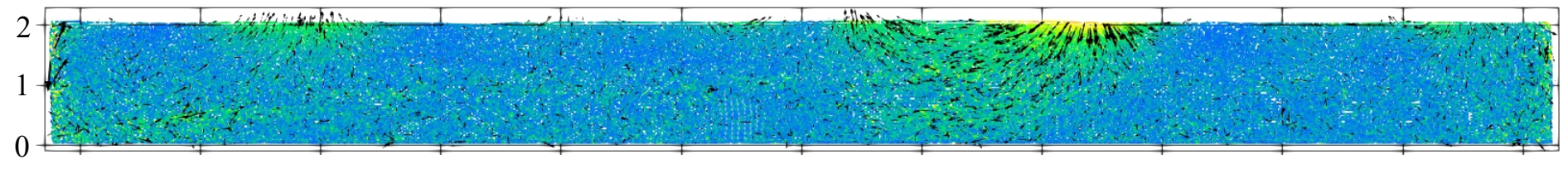

(c) $s=25$

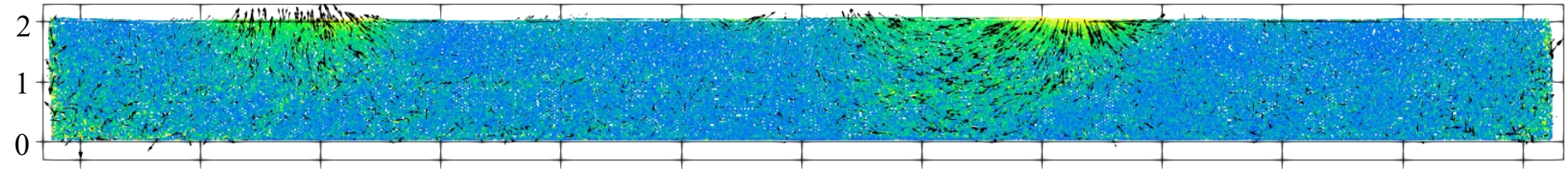

(d) $s=$ Inf.

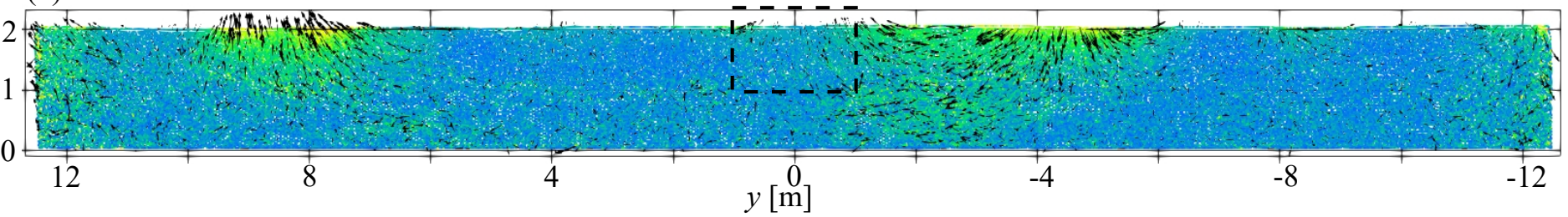




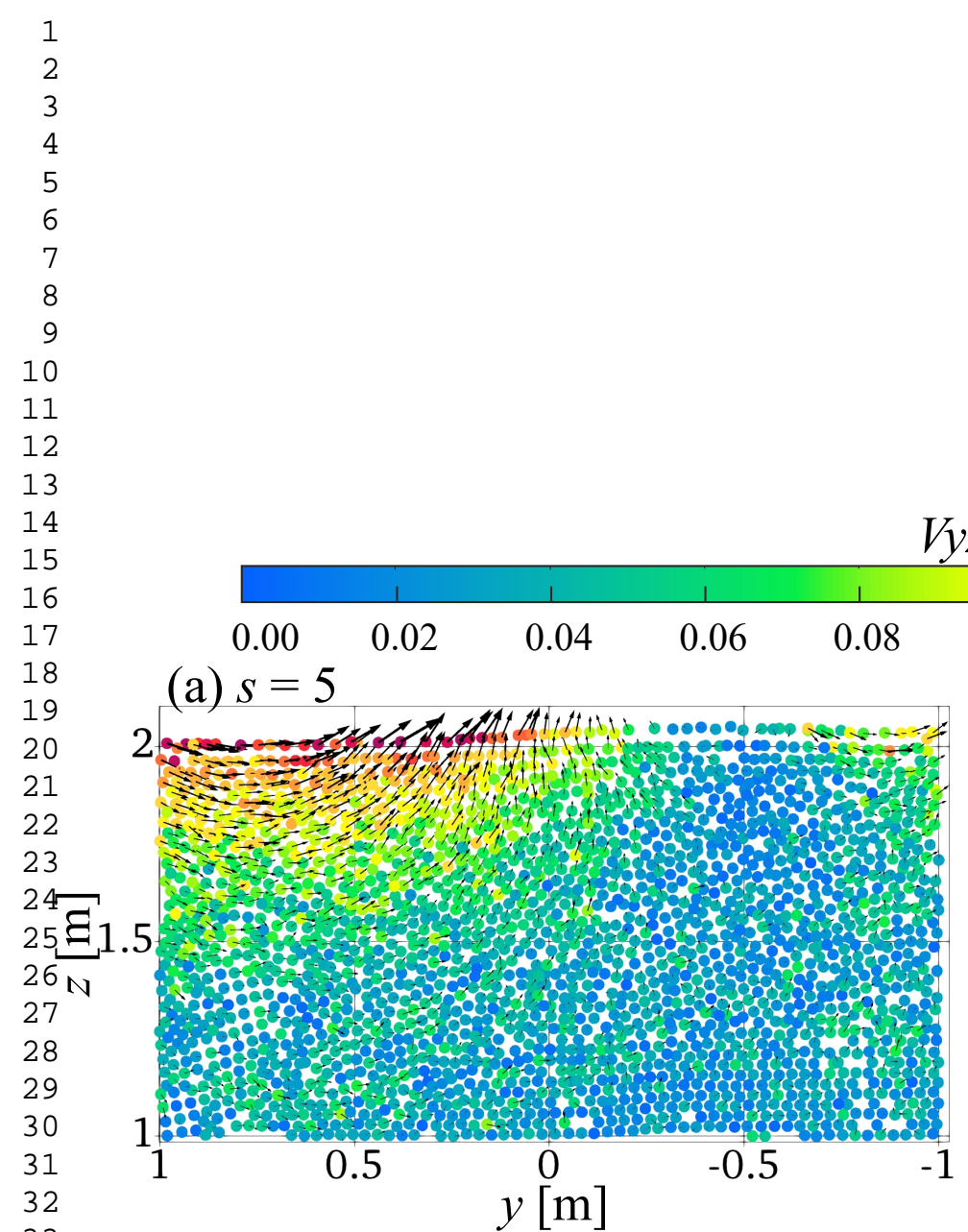
(c) $s=25$

$50^{\text {Figure 17: }}$ Snapshots of the velocity fields at the $Y Z$-plane where $x=0$, close to the centre of the FloWave tank $(x, y=0)$, as indicated by the black 51dashed square in Fig. 15, for Cases $1 \sim 4$ at $t=30 \mathrm{~s}$. 


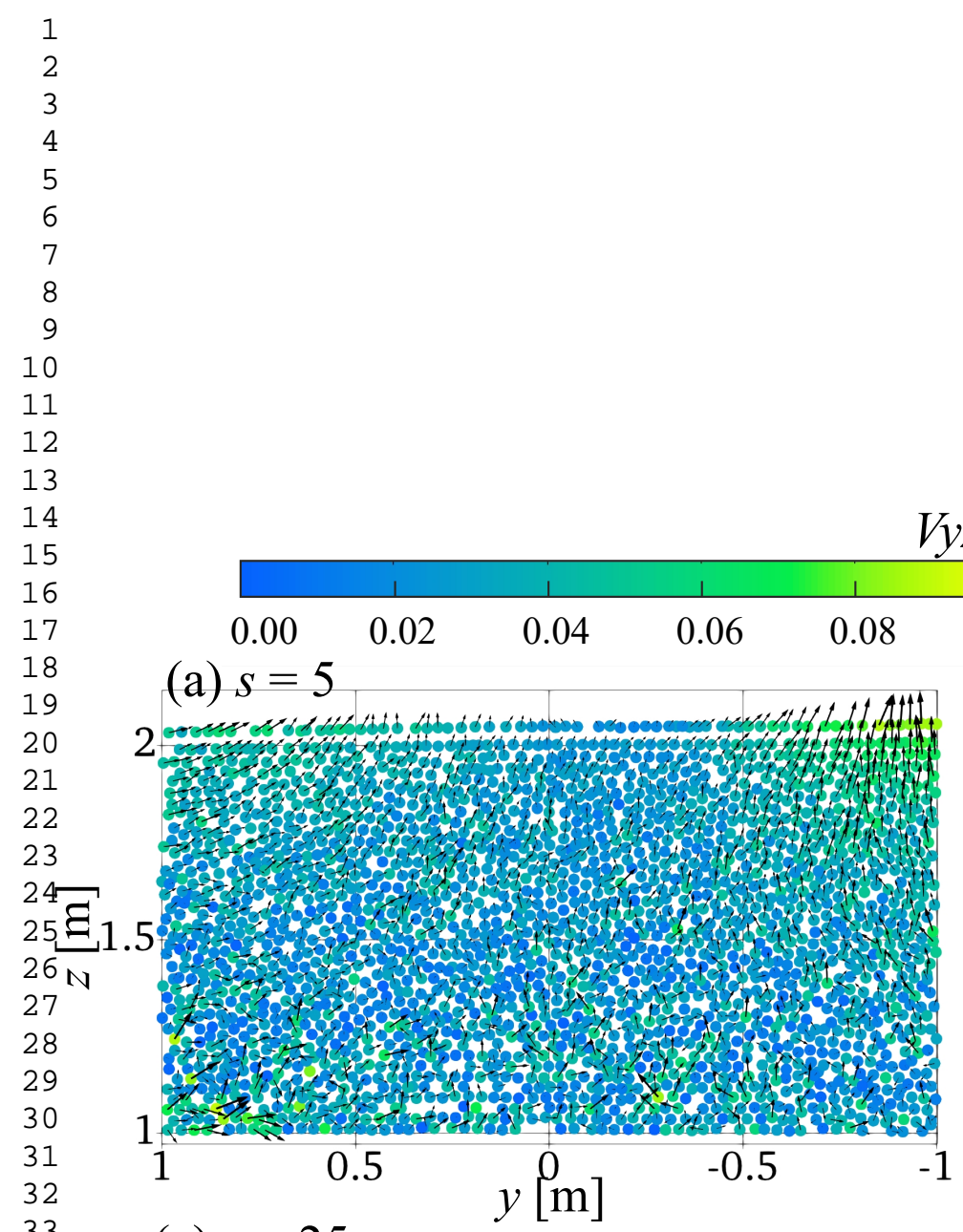

$V y z[\mathrm{~m} / \mathrm{s}]$

$\begin{array}{llllll}0.10 & 0.12 & 0.14 & 0.16 & 0.18 & 0.20\end{array}$

(c) $s=25$

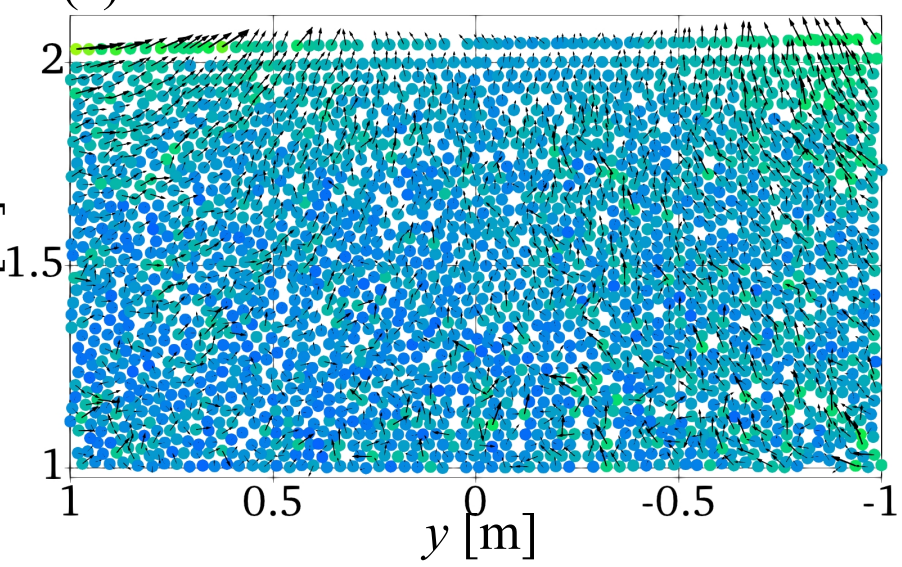

(b) $s=10$

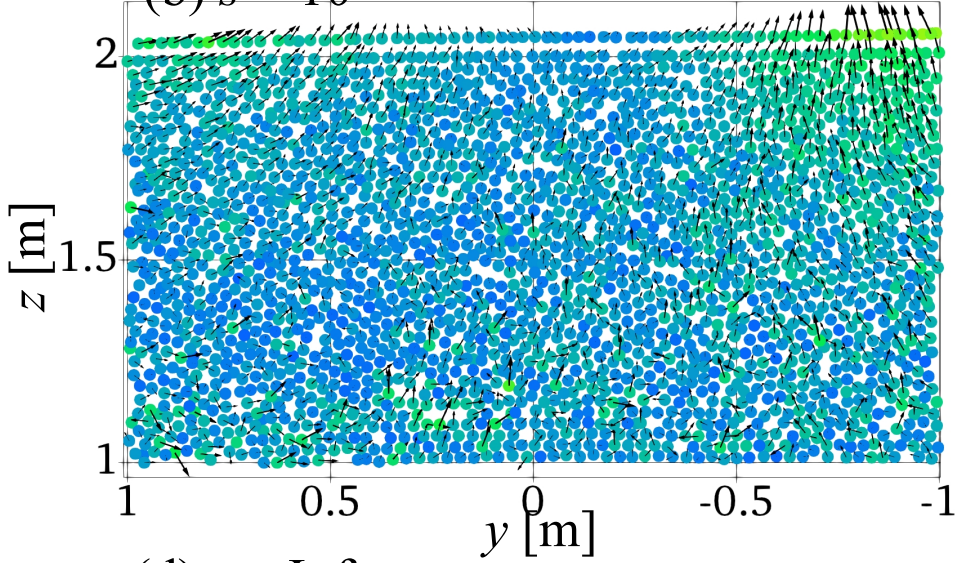

(d) $s=$ Inf.

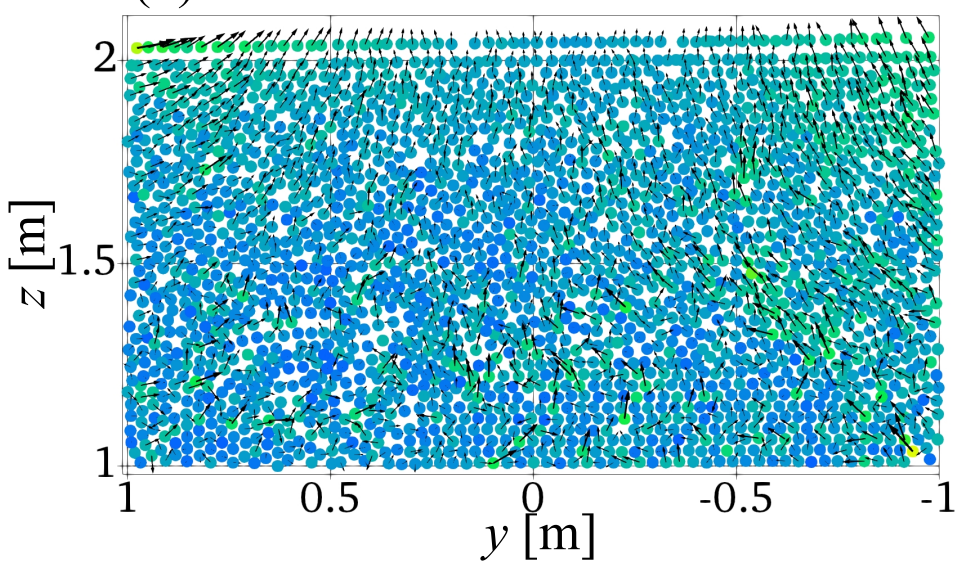

\section{9}

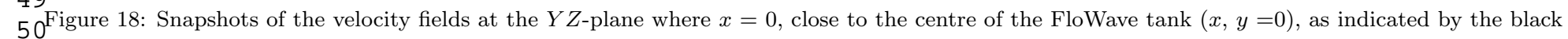
51dashed square in Fig. 16, for Cases $5 \sim 8$ at $t=30 \mathrm{~s}$. 
(a) $s=5$

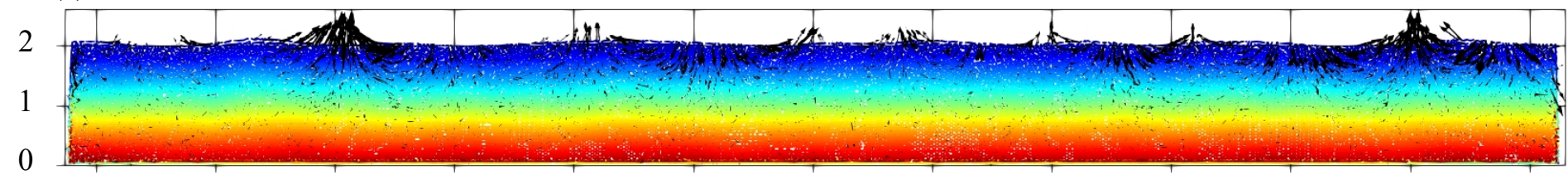

(b) $s=10$

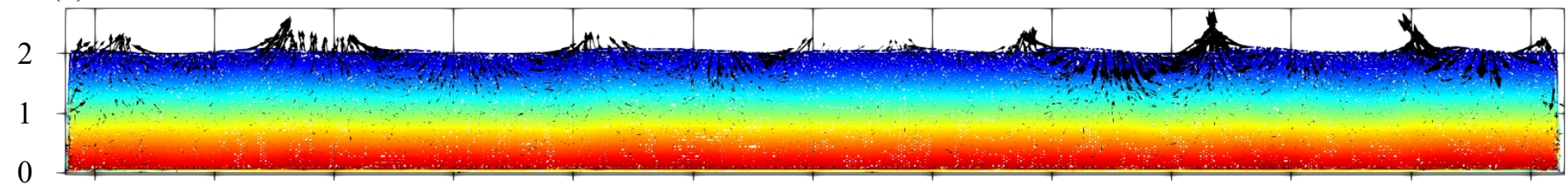

(c) $s=25$

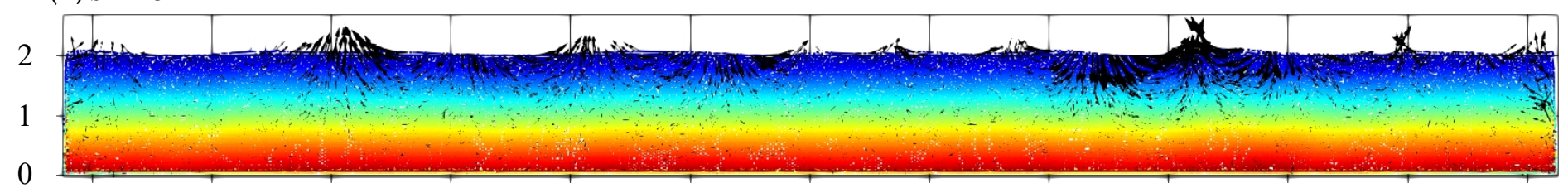

(d) $s=$ Inf.

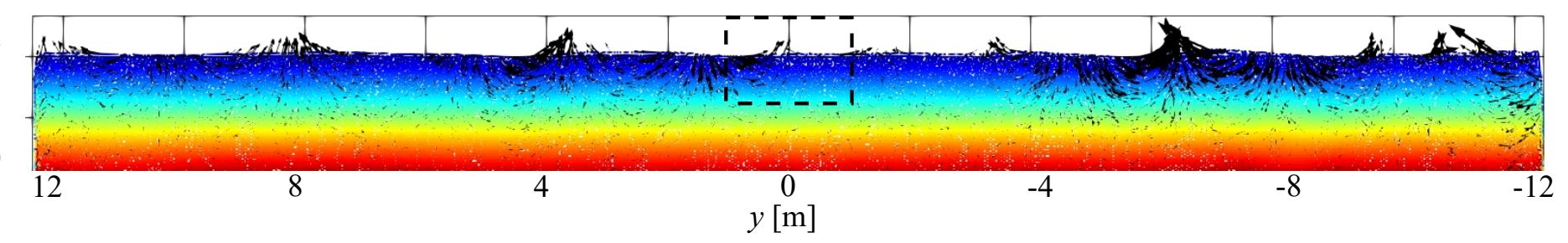

48 Figure 19: Snapshots of the pressure fields at the $Y Z$-plane where $x=0$, close to the centre of the FloWave tank $(x, y=0)$, as indicated by the black

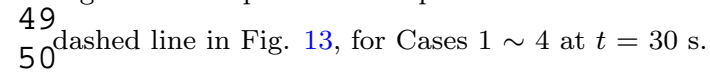


48Figure 20: Snapshots of the pressure fields at the $Y Z$-plane where $x=0$, close to the centre of the FloWave tank $(x, y=0)$, as indicated by the black

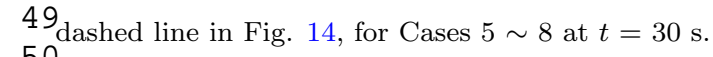




\begin{tabular}{|c|c|c|c|c|c|c|c|c|c|c|}
\hline \multicolumn{10}{|c|}{ Pressure $[\mathrm{Pa}]$} & \multirow{2}{*}{$\times 10^{4}$} \\
\hline & & 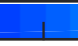 & $\perp$ & $\perp$ & $\perp$ & $\perp$ & $\perp$ & $\perp$ & $\perp$ & \\
\hline 0 & 0.2 & 0.4 & 0.6 & 0.8 & 1 & $\begin{array}{c}1.2 \\
\text { (b) } s\end{array}$ & $0^{1.4}$ & 1.6 & 1.8 & 2 \\
\hline
\end{tabular}

20

21

22

23

$24 £$

$26^{N}$

27

28

29

30

31

32

33

34

35

36

37

38

39

$40^{\circ}$

42

43

44

45

46

47

48

49

50Figure 21: Snapshots of the pressure fields at the $Y Z$-plane where $x=0$, close to the centre of the FloWave tank $(x, y=0)$, as indicated by the black 51dashed square in Fig. 19, for Cases $1 \sim 4$ at $t=30 \mathrm{~s}$.

52

53

54

55

56

57

58

59

60

61

62

63

64 


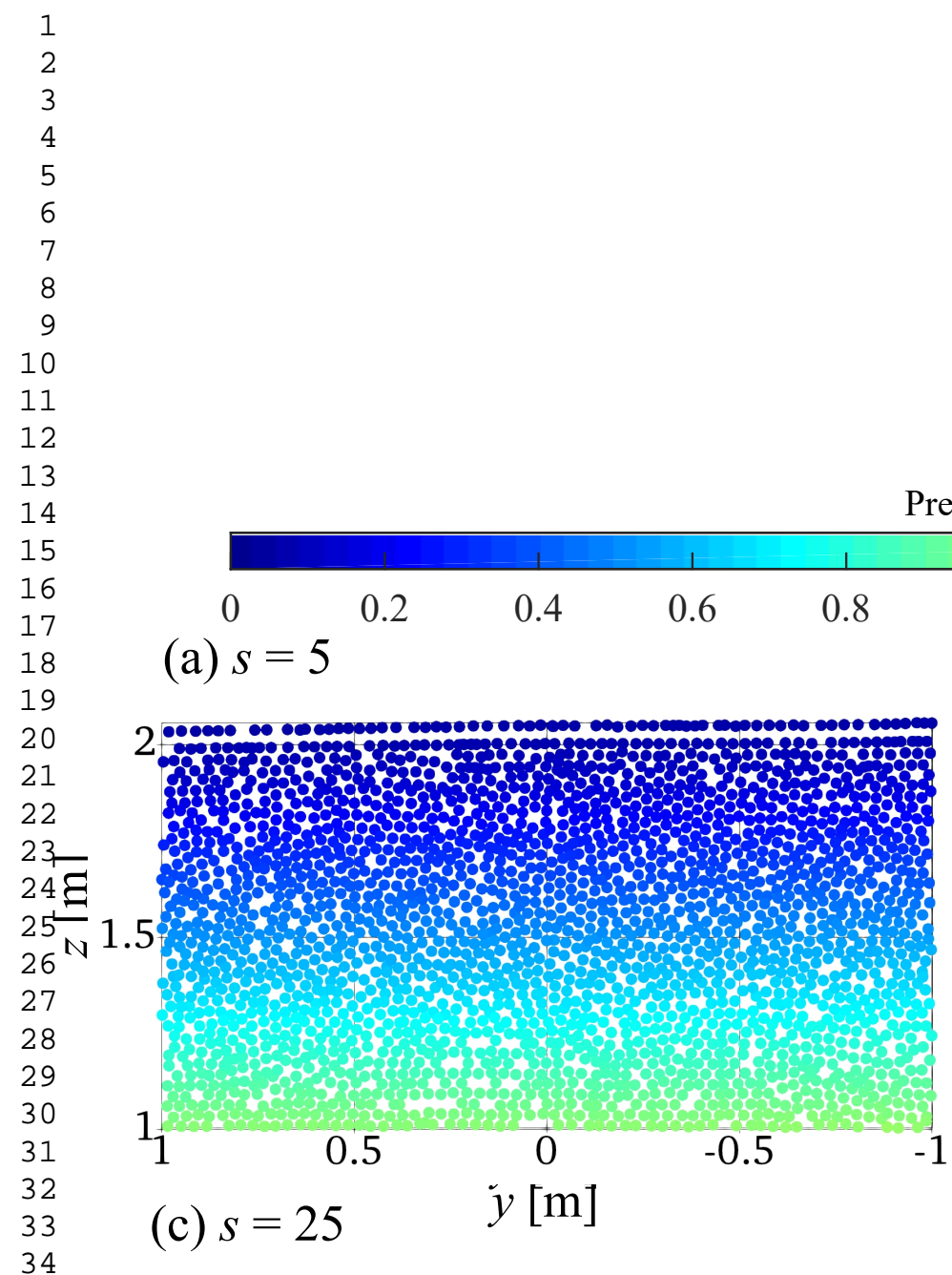

35

36

37

38

49巨

$41^{\mathrm{N}} 1.5$
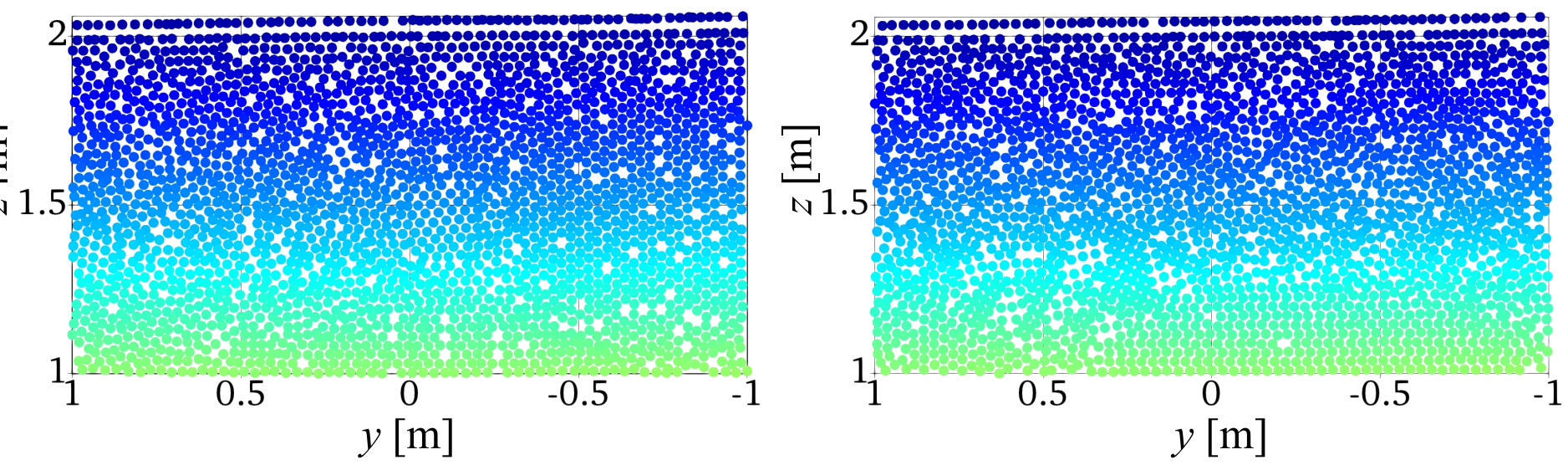

49

5๑Figure 22: Snapshots of the pressure fields at the $Y Z$-plane where $x=0$, close to the centre of the FloWave tank $(x, y=0)$, as indicated by the black 51dashed square in Fig. 20, for Cases $5 \sim 8$ at $t=30 \mathrm{~s}$. 


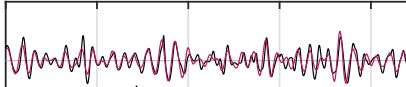

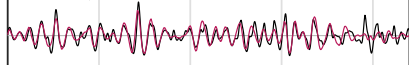

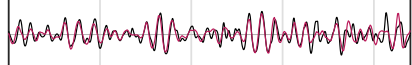

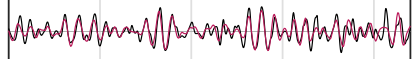

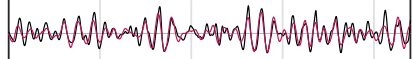

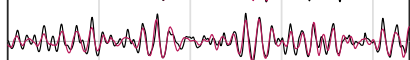

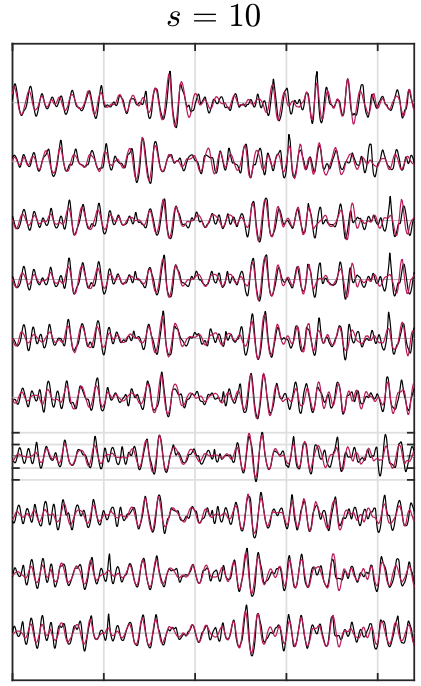
Time $[\mathrm{s}]$ 60

(a) $H_{m 0}=0.15 \mathrm{~m}$ and $T_{p}=1.5 \mathrm{~s}$

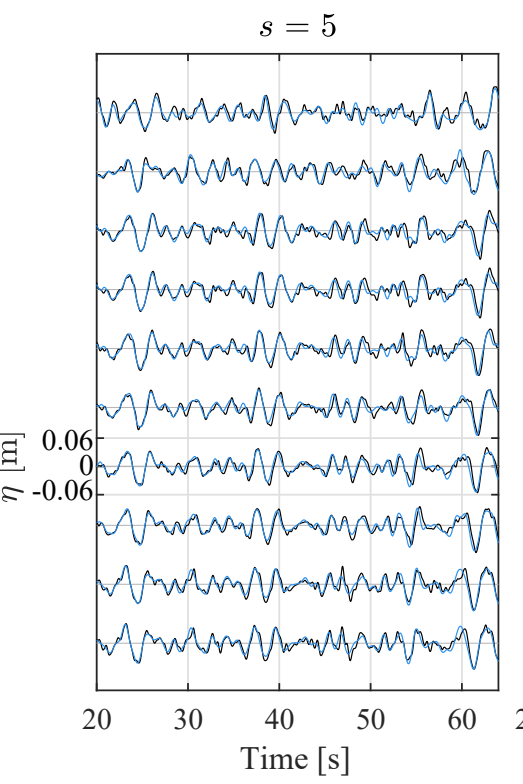

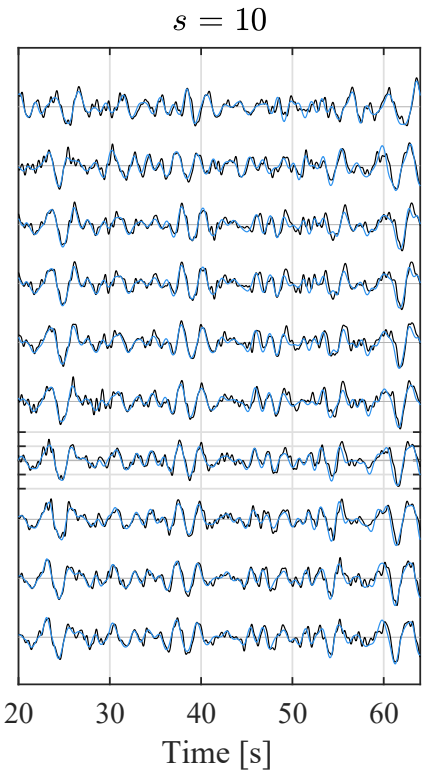

$s=25$

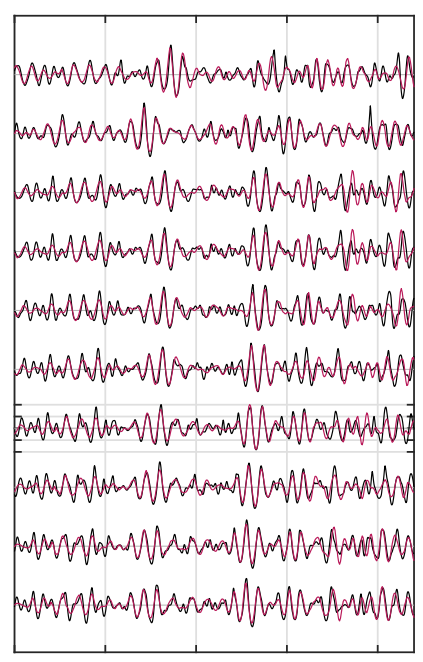

20

Time [s]

$s=$ Inf.

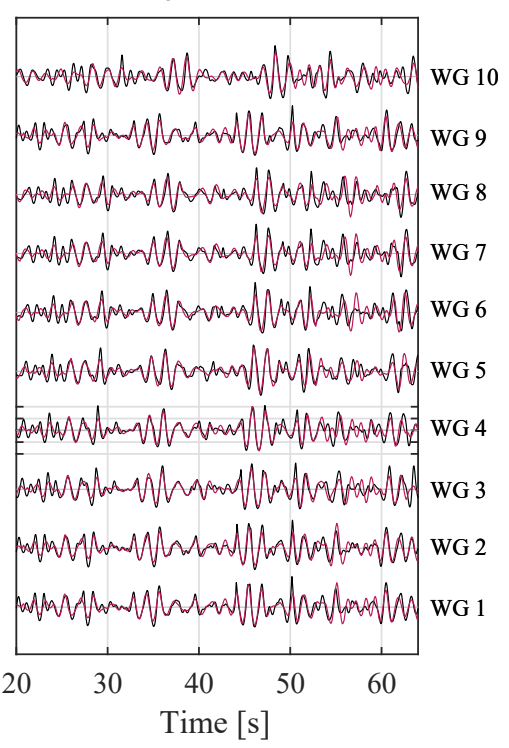

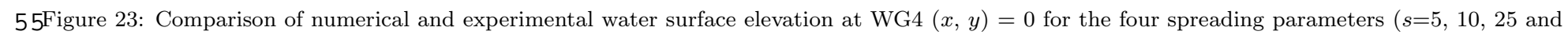
56Inf.), where (a) $H_{m 0}=0.15 \mathrm{~m}$ and $T_{p}=1.5 \mathrm{~s}$ and (b) $H_{m 0}=0.075 \mathrm{~m}$ and $T_{p}=3.0 \mathrm{~s}$.

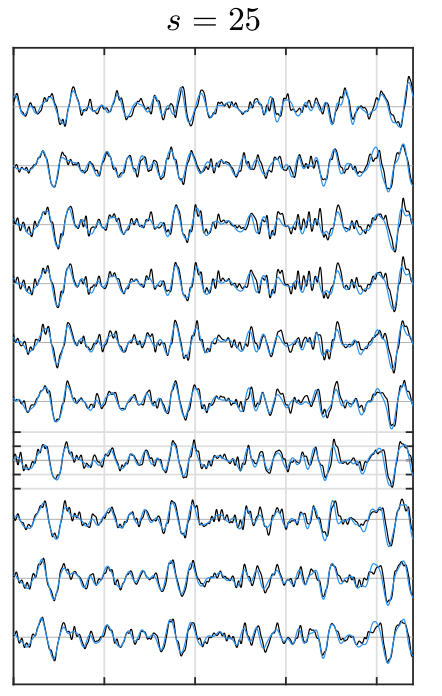

$\begin{array}{lllll}20 & 30 & 40 & 50 & 60\end{array}$

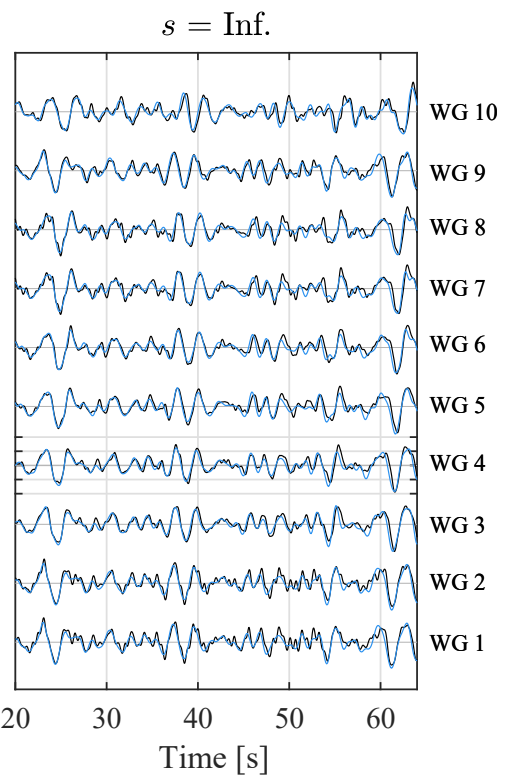




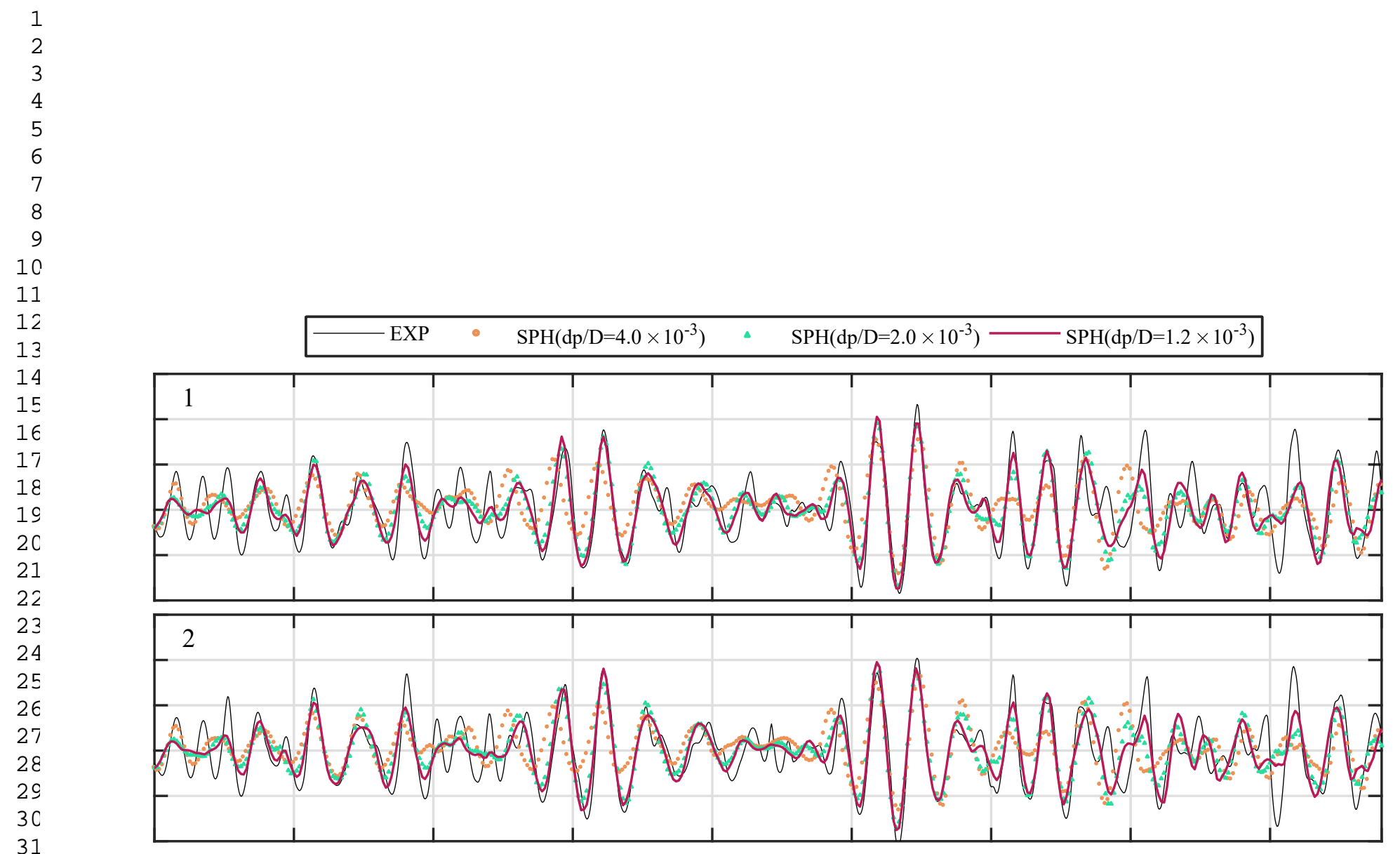

53Figure 24: Comparison of numerical and experimental water surface elevation at WG4 $(x, y)=0$ for the four spreading parameters $(s=5,10,25$ and

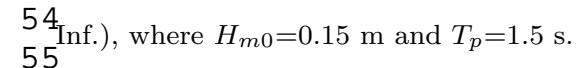




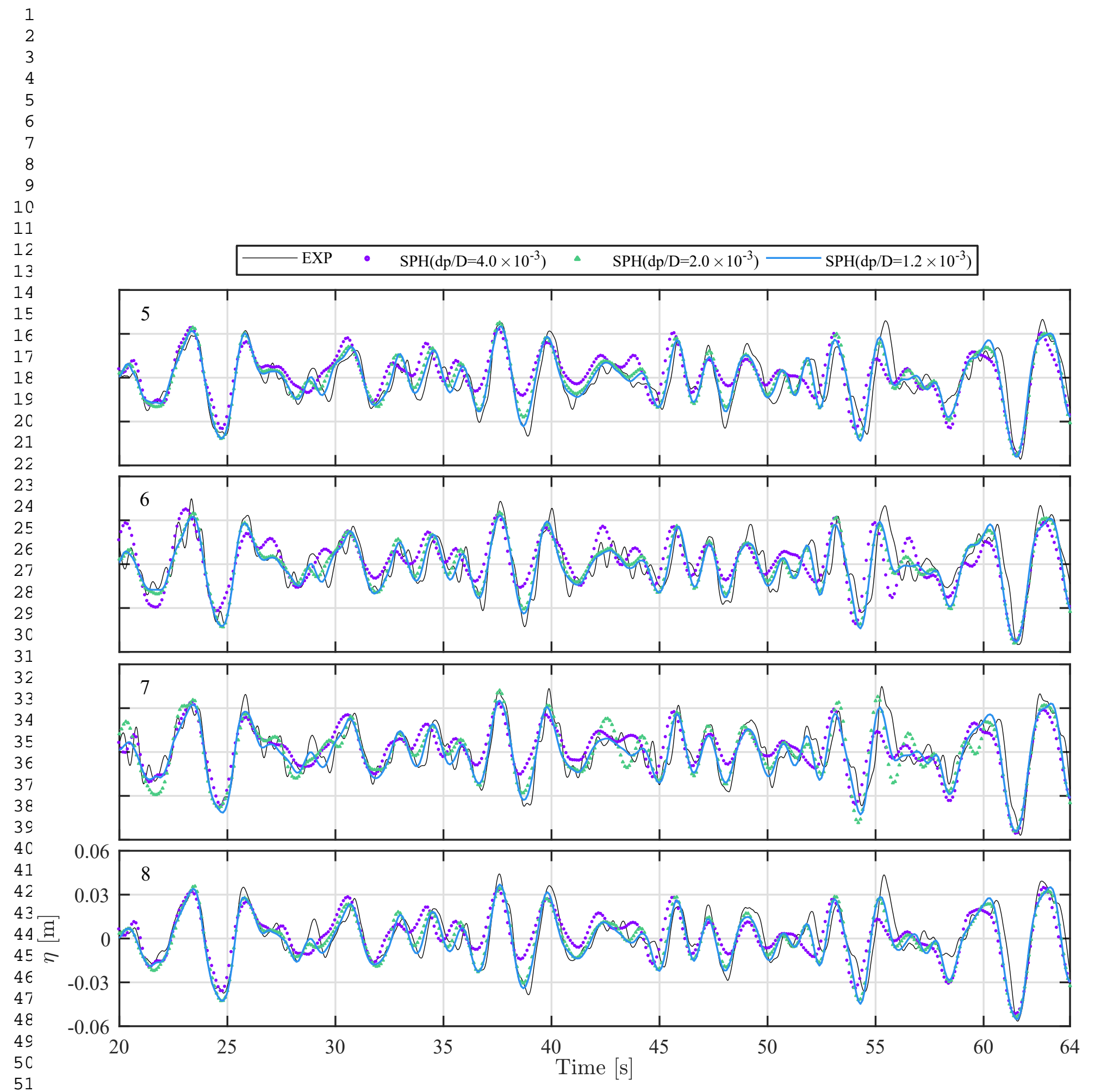

52

53Figure 25: Comparison of numerical and experimental water surface elevation at WG4 $(x, y)=0$ for the four spreading parameters $(s=5,10,25$ and $54_{\text {Inf.), }}$, where $H_{m 0}=0.075 \mathrm{~m}$ and $T_{p}=3.0 \mathrm{~s}$.

56

57

58 


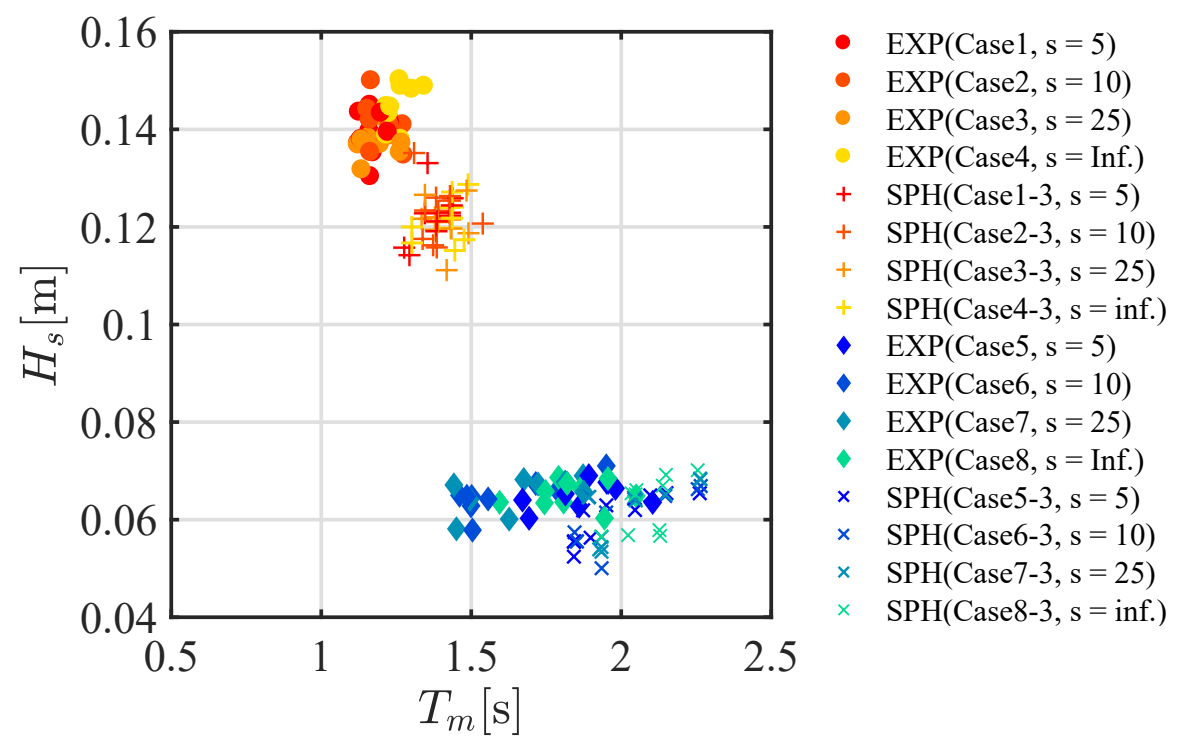

Figure 26: $H_{s}$ and $T_{m}$ combinations for all sea states with fine particle resolutions. 

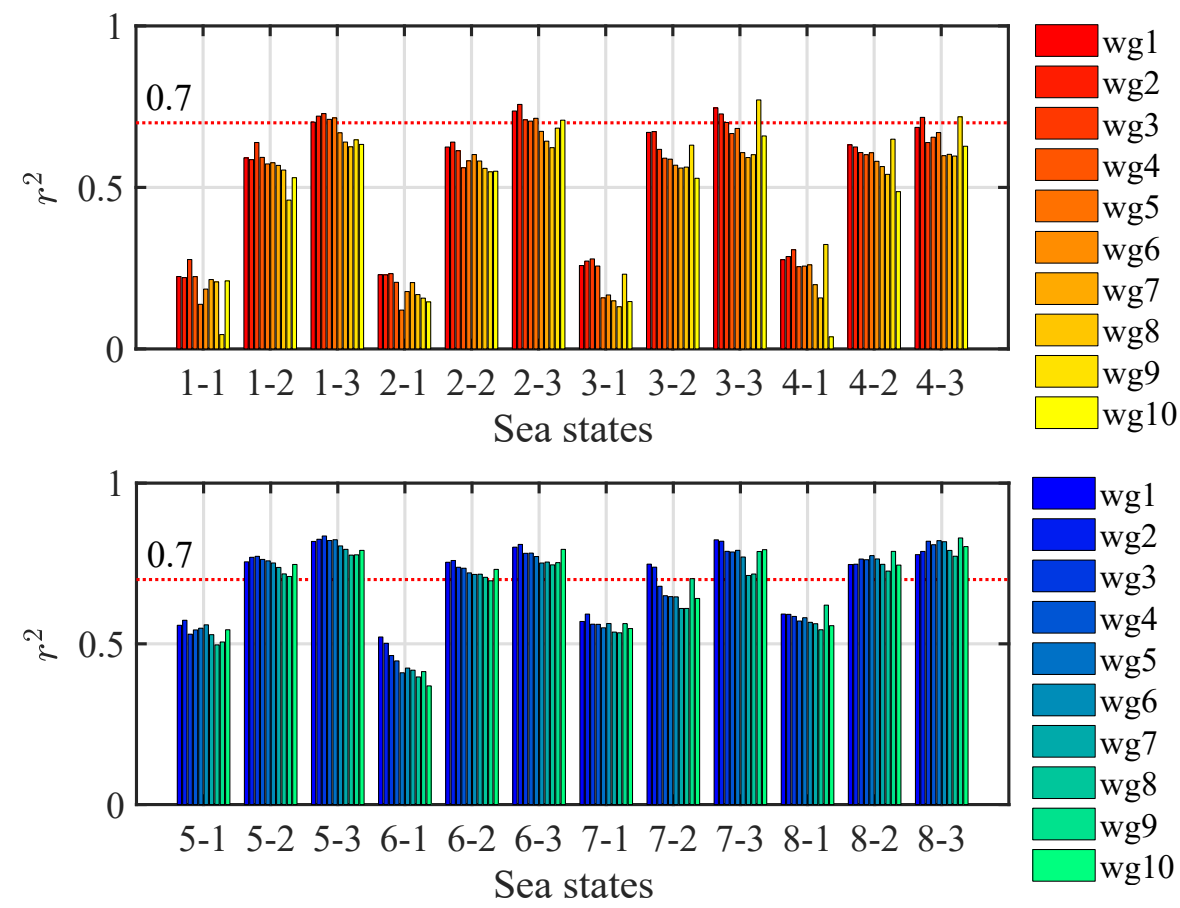

Figure 27: Coefficient of determination $\left(r^{2}\right)$ for all sea states at all gauges. The $x$-axis labels indicate the sea state-resolution combination, where 1 represents a coarse resolution and 3 represents a fine resolution. 


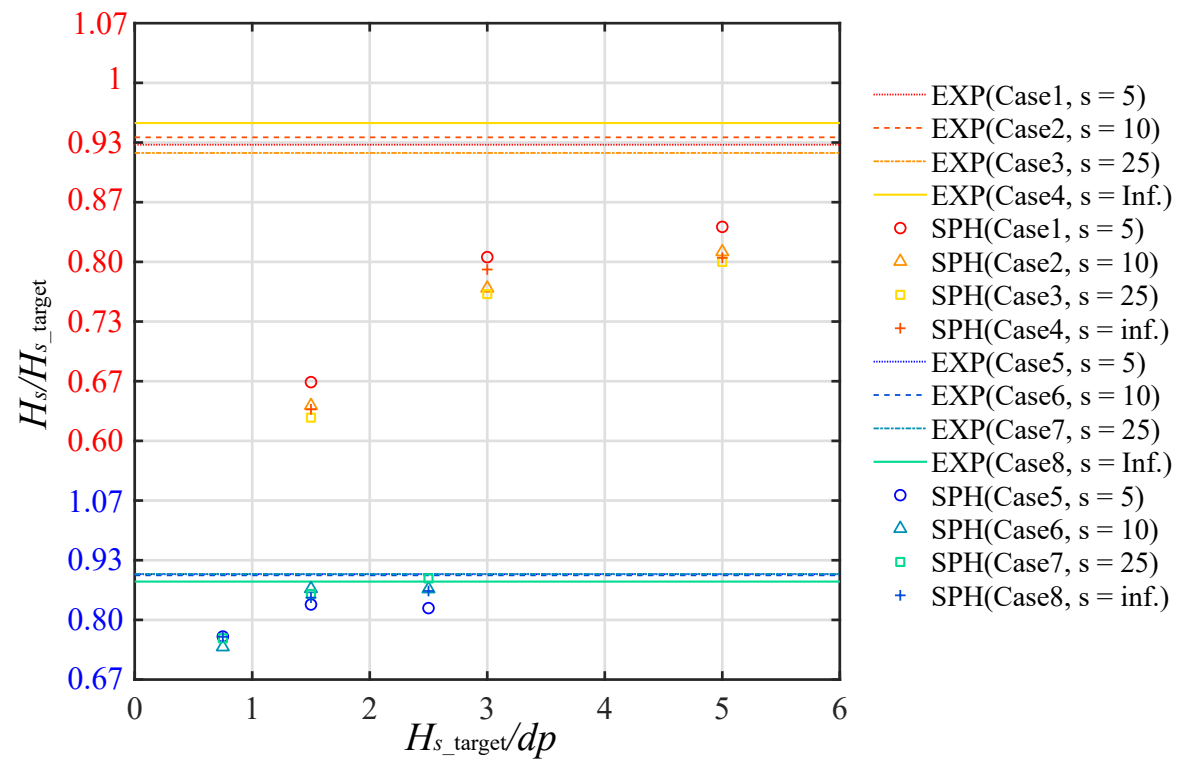

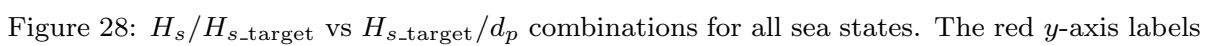
denote $H_{s} / H_{s_{-} \text {target }}$ for Cases $1 \sim 4$ and the blue labels denote Cases $5 \sim 8$. The warm and cold colors represent high-steepness $(0.043)$ and low-steepness $(0.0053)$, respectively. $H_{s}$ was calculated using surface elevations at WG4 $(x, y=0)$. 
Table 3: Error percentages (\%) for significant wave height between experimental and numerical results at WG4.

\begin{tabular}{c|ccc|ccc}
\hline & \multicolumn{3}{|c|}{ Cases 1 $\sim 4$} & \multicolumn{3}{c}{ Cases $5 \sim 8$} \\
\hline$s$ & Coarse & Middle & Fine & Coarse & Middle & Fine \\
\hline 5 & -28 & -13 & -10 & -15 & -7 & -8 \\
10 & -32 & -16 & -14 & -15 & -6 & -4 \\
25 & -31 & -16 & -12 & -18 & -4 & -4 \\
Inf. & -34 & -20 & -16 & -14 & -3 & +1 \\
\hline
\end{tabular}




\section{Appendix A. Experimental error}

Gauge calibration was carried out prior to testing using a three point calibration by moving the gauges to known positions and recording the measured voltages. Gauges were moved to $-0.1 \mathrm{~m}, 0.1 \mathrm{~m}$ and $0 \mathrm{~m}$ and a linear fit was applied between the measured voltages and the known positions to obtain zero and gain values to be applied for all of the subsequent measurements. To quantify the experimental error, the standard deviation between the calculated positions (from the calibration) and the known positions is used. The average error is calculated as $0.19 \%$ over the $0.1 \mathrm{~m}$ range, which corresponds to a standard deviation of $1.9 \times 10^{-4} \mathrm{~m}$. Over an entire day of testing (approx 8 hours) it is also known that some drift in the gain values occurs due to temperature changes, resulting in maximum errors again in the order of $10^{-4} \mathrm{~m}$. However, as the tests were carried out in a short space of time after the calibration the 'drift' errors in these experiments will be negligible.

\section{References}

[1] S. Xu, S. Wang, C. G. Soares, Review of mooring design for floating wave energy converters, Renewable and Sustainable Energy Reviews 111 (2019) 595 - 621. doi:https://doi.org/10.1016/j.rser.2019.05.027.

[2] P. Qian, B. Feng, H. Liu, X. Tian, Y. Si, D. Zhang, Review on configuration and control methods of tidal current turbines, Renewable and Sustainable Energy Reviews 108 (2019) 125 - 139. doi:https://doi.org/10.1016/ j.rser.2019.03.051.

[3] A. Pacheco, O. Ferreira, Hydrodynamic changes imposed by tidal energy converters on extracting energy on a real case scenario, Applied Energy 180 (2016) 369 - 385. doi :https ://doi .org/10.1016/j . apenergy . 2016 . 07. 132.

[4] F. Madhi, R. W. Yeung, On survivability of asymmetric wave-energy 
converters in extreme waves, Renewable Energy 119 (2018) 891 - 909. doi:https://doi.org/10.1016/j.renene.2017.07.123.

[5] G. Moretti, G. Pietro Rosati Papini, L. Daniele, D. Forehand, D. Ingram, R. Vertechy, M. Fontana, Modelling and testing of a wave energy converter based on dielectric elastomer generators, Proceedings of the Royal Society A: Mathematical, Physical and Engineering Sciences 475 (2222). doi: 10.1098/rspa.2018.0566.

[6] E. C. Moreno, P. Stansby, The 6-float wave energy converter m4: Ocean basin tests giving capture width, response and energy yield for several sites, Renewable and Sustainable Energy Reviews 104 (2019) 307 - 318. doi:https://doi.org/10.1016/j.rser.2019.01.033.

[7] M. Minoura, R. Takahashi, E. Okuyama, S. Naito, Generation of extreme wave composed of ring waves in a circular basin., in: Proceedings of the Nineteenth International Offshore and Polar Engineering Conference, 21-26 July, Osaka, Japan, International Society of Offshore and Polar Engineers, Osaka, Japan, 2009, pp. 389-396.

[8] K. Maeda, N. Hosotani, K. Tamura, H. Ando, Wave making properties of circular basin, in: Proceedings of the 2004 International Symposium on Underwater Technology (IEEE Cat. No.04EX869), 2004, pp. 349-354. doi:10.1109/UT.2004.1405603.

[9] D. Ingram, R. Wallace, A. Robinson, I. Bryden, The design and commissioning of the first, circular, combined current and wave test basin., in: Proceedings of Oceans 2014 MTS/IEEE Taipei, Taiwan, Institute of Electrical and Electronics Engineers (IEEE), United States, 2014.

[10] S. Draycott, D. Noble, T. Davey, T. Bruce, D. Ingram, L. Johanning, H. Smith, A. Day, Re-creation of site-specific multi-directional waves with non-collinear current, Ocean Engineering 152 (2018) 391-403. doi: $10.1016 / j$.oceaneng. 2017.10.047. 
[11] S. Draycott, T. Davey, D. Ingram, J. Lawrence, A. Day, L. Johanning, Using a phase-time-path-difference approach to measure directional wave spectra in flowave, in: Proceedings of the 11th European Wave and Tidal Energy Conference (EWTEC2015), 2015.

[12] S. Draycott, T. Davey, D. Ingram, A. Day, L. Johanning, The spair method: Isolating incident and reflected directional wave spectra in multidirectional wave basins, Coastal Engineeringdoi:10.1016/j.coastaleng.2016.04. 012.

[13] S. Draycott, J. Steynor, T. Davey, D. Ingram, Isolating incident and reflected wave spectra in the presence of current, Coastal Engineering Journaldoi: $10.1080 / 05785634.2017 .1418798$.

[14] D. R. Sutherland, D. R. Noble, J. Steynor, T. Davey, T. Bruce, Characterisation of current and turbulence in the flowave ocean energy research facility, Ocean Engineering 139 (2017) 103 - 115. doi:https: //doi.org/10.1016/j.oceaneng.2017.02.028.

[15] D. Sutherland, S. Ordonez-Sanchez, M. R. Belmont, I. Moon, J. Steynor, T. Davey, T. Bruce, Experimental optimisation of power for large arrays of cross-flow tidal turbines, Renewable Energy 116 (2018) 685 - 696. doi: https://doi.org/10.1016/j.renene.2017.10.011.

[16] R. Gabl, T. Davey, E. Nixon, J. Steynor, D. M. Ingram, Comparison of a floating cylinder with solid and water ballast, Water 11 (12). doi:10. 3390/w11122487.

URL https://www.mdpi.com/2073-4441/11/12/2487

[17] M. S. Turnbull, A. G. L. Borthwick, R. E. Taylor, Numerical wave tank based on a $\sigma$-transformed finite element inviscid flow solver, International Journal for Numerical Methods in Fluids 42 (6) (2013) 641-663. doi: $10.1002 / f l d .539$. 
[18] X.-Z. Zhao, Validation of a cip-based tank for numerical simulation of free surface flows, Acta Mechanica Sinica 27 (6) (2011) 877-890. doi:10.1007/ s10409-011-0510-4.

[19] P. Higuera, I. J. Losada, J. L. Lara, Three-dimensional numerical wave generation with moving boundaries, Coastal Engineering 101 (2015) 35 47. doi:https://doi.org/10.1016/j.coastaleng.2015.04.003.

[20] P. Higuera, J. L. Lara, I. J. Losada, Three-dimensional interaction of waves and porous coastal structures using openfoam $囚$. part i: Formulation and validation, Coastal Engineering 83 (2014) 243 - 258. doi:https://doi. org/10.1016/j. coastaleng. 2013.08.010.

[21] W. Christian, D. Josh, A. Benazzou, R. J. V., Performance assessment of the overset grid method for numerical wave tank experiments in the openfoam environment, in: Proceedings of the ASME 2018 37th International Conference on Ocean, Offshore and Arctic Engineering, Madrid, Spain, 2018. doi:10.1115/OMAE2018-77564.

[22] R. A. Gingold, J. J. Monaghan, Smoothed particle hydrodynamics: theory and application to non-spherical stars, Monthly Notices of the Royal Astronomical Society 181 (3) (1977) 375-389. doi:10.1093/mnras/181.3.375.

[23] S. KOSHIZUKA, A particle method for incompressible viscous flow with fluid fragmentation, Comput. Fluid Dyn. J. 4 (1995) 29-46.

URL https://ci.nii.ac.jp/naid/10006968931/en/

[24] H. Gotoh, A. Khayyer, On the state-of-the-art of particle methods for coastal and ocean engineering, Coastal Engineering Journal 60 (1) (2018) 79-103. arXiv:https://doi .org/10.1080/21664250. 2018. 1436243, doi:10.1080/21664250.2018.1436243.

[25] X. Cao, F. Ming, A. Zhang, L. Tao, Multi-phase sph modelling of air effect on the dynamic flooding of a damaged cabin, Computers Fluids 163 (2018) 7 - 19. doi:https://doi.org/10.1016/j.compfluid.2017.12.012. 
URL http://www.sciencedirect.com/science/article/pii/ S0045793017304504

[26] F. Ming, A. Zhang, H. Cheng, P. Sun, Numerical simulation of a damaged ship cabin flooding in transversal waves with smoothed particle hydrodynamics method, Ocean Engineering 165 (2018) 336 - 352. doi:https://doi.org/10.1016/j.oceaneng.2018.07.048.

URL http://www.sciencedirect.com/science/article/pii/ S0029801818304049

[27] A. Trimulyono, H. Hashimoto, Experimental validation of smoothed particle hydrodynamics on generation and propagation of water waves, Journal of Marine Science and Engineering 7 (1). doi:10.3390/jmse7010017.

URL https://www.mdpi.com/2077-1312/7/1/17

[28] P. Sun, A. Colagrossi, S. Marrone, M. Antuono, A. Zhang, Multiresolution delta-plus-sph with tensile instability control: Towards high reynolds number flows, Computer Physics Communications 224 (2018) 63 - 80. doi:https://doi.org/10.1016/j.cpc.2017.11.016.

URL http://www.sciencedirect.com/science/article/pii/ S0010465517303995

[29] M. D. Green, J. Peiró, Long duration sph simulations of sloshing in tanks with a low fill ratio and high stretching, Computers Fluids 174 (2018) 179 - 199. doi:https://doi.org/10.1016/j.compfluid.2018.07.006.

URL http://www.sciencedirect.com/science/article/pii/ S0045793018303979

[30] S. Shao, E. Y. Lo, Incompressible sph method for simulating newtonian and non-newtonian flows with a free surface, Advances in Water Resources 26 (7) (2003) 787 - 800. doi:https: //doi.org/10.1016/S0309-1708(03)00030-7.

URL http://www.sciencedirect.com/science/article/pii/ S0309170803000307 
[31] D. Molteni, A. Colagrossi, A simple procedure to improve the pressure evaluation in hydrodynamic context using the sph, Computer Physics Communications 180 (6) (2009) 861 - 872. doi:https://doi.org/10.1016/j. cpc. 2008.12.004.

[32] A. Crespo, J. Domínguez, B. Rogers, M. Gómez-Gesteira, S. Longshaw, R. Canelas, R. Vacondio, A. Barreiro, O. García-Feal, Dualsphysics: Opensource parallel cfd solver based on smoothed particle hydrodynamics (sph), Computer Physics Communications 187 (2015) 204 - 216. doi:https: //doi.org/10.1016/j.cpc.2014.10.004.

[33] C. Altomare, A. Crespo, B. Rogers, J. Dominguez, X. Gironella, M. GómezGesteira, Numerical modelling of armour block sea breakwater with smoothed particle hydrodynamics, Computers \& Structures 130 (2014) 3445. doi:https://doi.org/10.1016/j.compstruc.2013.10.011.

[34] A. Crespo, C. Altomare, J. Domínguez, J. González-Cao, M. GómezGesteira, Towards simulating floating offshore oscillating water column converters with smoothed particle hydrodynamics, Coastal Engineering 126 (2017) 11 -26. doi:https://doi.org/10.1016/j.coastaleng.2017.05. 001.

[35] R. Canelas, M. Brito, O. Feal, J. Domínguez, A. Crespo, Extending dualsphysics with a differential variational inequality: modeling fluidmechanism interaction, Applied Ocean Research 76 (2018) 88 - 97. doi: https://doi.org/10.1016/j.apor.2018.04.015.

[36] D. F. Gunn, M. Rudman, R. C. Cohen, Wave interaction with a tethered buoy: Sph simulation and experimental validation, Ocean Engineering 156 (2018) 306 - 317. doi:https://doi.org/10.1016/j.oceaneng. 2018. 03.001.

URL http://www.sciencedirect.com/science/article/pii/ S0029801818302324 
[37] M. Masuda, Y. Sasahara, K. Minami, T. Tezdogan, A. Incecik, Use of the mps method to estimate the energy conversion efficiency of the owc-wec (first report), Ocean Engineering 188 (2019) 106133. doi:https://doi.org/10.1016/j.oceaneng.2019.106133.

URL http://www.sciencedirect.com/science/article/pii/ S002980181930321X

[38] M. Brito, R. Canelas, O. García-Feal, J. Domínguez, A. Crespo, R. Ferreira, M. Neves, L. Teixeira, A numerical tool for modelling oscillating wave surge converter with nonlinear mechanical constraints, Renewable Energy 146 (2020) 2024 - 2043. doi:https://doi.org/10.1016/j.renene.2019.08.034. URL http://www.sciencedirect.com/science/article/pii/ S0960148119312212

[39] T. Kanehira, H. Mutsuda, Y. Doi, N. Taniguchi, S. Draycott, D. Ingram, Development and experimental validation of a multidirectional circular wave basin using smoothed particle hydrodynamics, Coastal Engineering Journal 61 (1) (2019) 109-120. doi:10.1080/21664250 .2018.1560922.

[40] D. R. Fuhrman, P. A. Madsen, H. B. Bingham, Numerical simulation of lowest-order short-crested wave instabilities, Journal of Fluid Mechanics 563 (2006) 415-441. doi:10.1017/S0022112006001236.

[41] X. Ren, N. Mizutani, T. Nakamura, Development of a numerical circular wave basin based on the two-phase incompressible flow model, Ocean Engineering 101 (2015) 93 - 100. doi:https: //doi.org/10.1016/j.oceaneng. 2015.04.003.

URL http://www.sciencedirect.com/science/article/pii/ S0029801815000785

[42] Z. Wei, R. A. Dalrymple, M. Xu, R. Garnier, M. Derakhti, Short-crested waves in the surf zone, Journal of Geophysical Research: Oceans 122 (5) (2017) 4143-4162. doi:10.1002/2016JC012485. 
[43] A. Hérault, G. Bilotta, R. A. Dalrymple, Sph on gpu with cuda, Journal of Hydraulic Research 48 (sup1) (2010) 74-79. doi:10.1080/00221686. 2010.9641247.

[44] R. A. Dalrymple, A mechanism for rip current generation on an open coast, Journal of Geophysical Research (1896-1977) 80 (24) (1975) 3485-3487. doi:10.1029/JC080i024p03485.

[45] J. T. Kirby, M. Derakhti, Short-crested wave breaking, European Journal of Mechanics - B/Fluids 73 (2019) 100 - 111, breaking Waves. doi:https://doi.org/10.1016/j.euromechflu.2017.11.001.

URL http://www.sciencedirect.com/science/article/pii/ S0997754617302923

[46] F. Shi, J. T. Kirby, J. C. Harris, J. D. Geiman, S. T. Grilli, A high-order adaptive time-stepping tvd solver for boussinesq modeling of breaking waves and coastal inundation, Ocean Modelling 43-44 (2012) 36 - 51. doi:https://doi.org/10.1016/j.ocemod.2011.12.004.

URL http://www.sciencedirect.com/science/article/pii/ S1463500311002010

[47] W. J. Pierson Jr., L. Moskowitz, A proposed spectral form for fully developed wind seas based on the similarity theory of s. a. kitaigorodskii, Journal of Geophysical Research 69 (24) (1964) 5181-5190. doi: 10.1029/JZ069i024p05181.

[48] M. D. Miles, E. R. Funke, A comparison of methods for synthesis of directional seas, Journal of Offshore Mechanics and Arctic Engineering 111. doi:0.1115/1.3257137.

[49] H. Wendland, Piecewise polynomial, positive definite and compactly supported radial functions of minimal degree, Advances in Computational Mathematics 4 (1) (1995) 389-396. doi:10.1007/BF02123482. 
[50] R. Dalrymple, B. Rogers, Numerical modeling of water waves with the sph method, Coastal Engineering 53 (2) (2006) 141 - 147, coastal Hydrodynamics and Morphodynamics. doi:https://doi.org/10.1016/j. coastaleng. 2005.10.004.

[51] E. Y. Lo, S. Shao, Simulation of near-shore solitary wave mechanics by an incompressible sph method, Applied Ocean Research 24 (5) (2002) 275 286. doi:https://doi.org/10.1016/S0141-1187(03)00002-6.

[52] H. Gotoh, Sub-particle-scale turbulence model for the mps methodlagrangian flow model for hydraulic engineering-, Computational Fluid Dynamics Jour. 9 (4) (2001) 339-347.

URL https://ci.nii.ac.jp/naid/10010349955/

[53] J. J. Monaghan, Smoothed particle hydrodynamics, Annual Review of Astronomy and Astrophysics 30 (1) (1992) 543-574. arXiv:https://doi. org/10.1146/annurev.aa.30.090192.002551, doi:10.1146/annurev . aa. 30.090192 .002551 .

URL https://doi.org/10.1146/annurev.aa.30.090192.002551

[54] R. A. Dalrymple, O. Knio, SPH Modelling of Water Waves, 2001, pp. $779-$ 787.

[55] M. Gómez-Gesteira, R. A. Dalrymple, Using a three-dimensional smoothed particle hydrodynamics method for wave impact on a tall structure, Journal of Waterway, Port, Coastal, and Ocean Engineering 130 (2) (2004) 63-69.

[56] A. Crespo, M. Gómez-Gesteira, R. Dalrymple, 3d sph simulation of large waves mitigation with a dike, Journal of Hydraulic Research 45 (5) (2007) 631-642. arXiv:https://doi.org/10.1080/00221686.2007.9521799, doi: 10.1080/00221686.2007.9521799.

URL https://doi.org/10.1080/00221686.2007.9521799

[57] J. Monaghan, J. Kajtar, Sph particle boundary forces for arbitrary boundaries, Computer Physics Communications 180 (10) (2009) 1811 - 
1820. doi:https://doi.org/10.1016/j.cpc.2009.05.008.

URL http://www.sciencedirect.com/science/article/pii/ S0010465509001544

[58] M. Ferrand, D. R. Laurence, B. D. Rogers, D. Violeau, C. Kassiotis, Unified semi-analytical wall boundary conditions for inviscid, laminar or turbulent flows in the meshless sph method, International Journal for Numerical Methods in Fluids 71 (4) (2012) 446-472. arXiv: https://onlinelibrary.wiley.com/doi/pdf/10.1002/fld.3666, doi: $10.1002 / f l d .3666$.

URL https://onlinelibrary.wiley.com/doi/abs/10.1002/fld.3666

[59] A. Crespo, M. Gómez-Gesteira, R. Dalrymple, Boundary conditions generated by dynamic particles in sph methods, Computers, Materials and Continua 5 (3) (2007) 173-184.

[60] A. Crespo, M. Gómez-Gesteira, R. Dalrymple, Boundary conditions generated by dynamic particles in sph methods, Computers, Materials and Continua 5 (3) (2007) 173-184.

[61] C. Altomare, J. Domínguez, A. Crespo, J. González-Cao, T. Suzuki, M. Gómez-Gesteira, P. Troch, Long-crested wave generation and absorption for sph-based dualsphysics model, Coastal Engineering 127 (2017) 37-54. doi:10.1016/j. coastaleng. 2017.06.004. 